\title{
Exterior Differential Systems with Symmetry
}

\author{
I.M. Anderson and M. E. Fels \\ Department of Mathematics and Statistics, Utah State University,Logan Utah, \\ USA, 84321, (anderson@math.usu.edu, fels@math.usu.edu)
}

Sept. 15, 2004

\begin{abstract}
We use the theory of reduction of exterior differential systems with symmetry to study the problem of using a symmetry group of a differential equation to find non-invariant solutions.
\end{abstract}

Keywords: Exterior Differential Systems, Symmetry

\section{Introduction}

One of Lie's fundamental contributions to the analysis of differential equations was to use a symmetry group of a differential equation to help find particular solutions. The solutions which are fixed under the action of the group are known as group invariant solutions and the mechanism for determining these particular solutions is well understood. At the other extreme lie solutions which have little or no symmetry. In order to find this type of solution Lie hoped to find a "reduced" system of differential equations (the resolvent in (Osvianikov, 1982)) whose solutions could be used to generate solutions without symmetry for the original equation.

Numerous problems arise with this approach to reduction when formulated within the usual jet space description of differential equations. Exterior differential systems (EDS) provide a more intrinsic geometric approach to studying differential equations and by translating Lie's reduction method into an EDS setting many of the problems in Lie's original approach disappear.

We associate to a differential equation with symmetry an exterior differential system with symmetry whose integral manifolds contain the solutions to the differential equation. The quotient or reduced exterior differential system by the symmetry group is then easy to define. We find that if the symmetry group of the EDS satisfies certain transversality conditions then the entire orbit of a solution to the original system of differential equations corresponds to a single integral manifold for the reduced EDS. Conversely, given a single integral manifold to the reduced EDS it is a simple matter to reconstruct solutions to the original system and these solutions form an orbit of non-invariant solutions.

(C) 2004 Kluwer Academic Publishers. Printed in the Netherlands. 


\section{Differential Equations and Symmetry}

We begin by reviewing a few basic definitions and properties of group actions and then apply these to the case of group actions on solution spaces of differential equations. Let $G$ be group and $\mathcal{S}$ a set. A left action of the group $G$ on the set $\mathcal{S}$ is a function $\mu: G \times \mathcal{S} \rightarrow \mathcal{S}$ that satisfies

$$
\begin{array}{ll}
\text { i] } \quad \mu(e, x)=x, \\
\text { ii] } \quad \mu(a, \mu(b, x))=\mu(a b, x), \quad a, b \in G, x \in \mathcal{S}, e=\text { identity. }
\end{array}
$$

For convenience we will often write $\mu(a, x)=a x$. Let $P(\mathcal{S})$ be the group of bijections of $\mathcal{S}$. For fixed $a \in G$, the function $\mu_{a}: \mathcal{S} \rightarrow \mathcal{S}$ given by $\mu_{a}(x)=\mu(a, x)$ is a permutation of $\mathcal{S}$. The map $G \rightarrow P(\mathcal{S})$ given by $a \rightarrow \mu_{a}$ is a group homomorphism from $G \rightarrow P(\mathcal{S})$. The orbit of a point $x \in \mathcal{S}$ is the subset $\mathcal{O}_{x} \subset \mathcal{S}$ given by

$$
\mathcal{O}_{x}=\{y \in \mathcal{S} \mid y=\mu(a, x) \text { for some } a \in G\} .
$$

The totality of orbits partition $\mathcal{S}$. This, in turn, defines an equivalence relation on $\mathcal{S}$ for which two points $x, y \in \mathcal{S}$ are equivalent if $\mathcal{O}_{x}=\mathcal{O}_{y}$. The set of equivalence classes are denoted by $\mathcal{S} / G$ and is called the quotient space of $\mathcal{S}$ by $G$, or the orbit space for the action of $G$ on $\mathcal{S}$. Let

$$
\mathbf{q}: \mathcal{S} \rightarrow \mathcal{S} / G
$$

be the projection $\mathbf{q}(x)=\mathcal{O}_{x}$. For each $x \in \mathcal{S}$ the isotropy subgroup, or stabilizer subgroup $G_{x} \subset G$ is defined by

$$
G_{x}=\{a \in G \mid \mu(a, x)=x\} .
$$

We think of $G_{x}$ as the symmetry group of the point $x \in \mathcal{S}$. The fixed point set $\mathcal{S}^{G}$ is

$$
\mathcal{S}^{G}=\{x \in \mathcal{S} \mid a x=x \quad \text { for all } a \in G\},
$$

and so, for $x \in \mathcal{S}^{G}, G_{x}=G$. A point $x \in \mathcal{S}$ with maximal symmetry will be in $\mathcal{S}^{G}$ while a point with no symmetry will have $G_{x}=\{e\}$.

Let $\Delta=0$ be a system of differential equations, and let $\mathcal{S}$ be the solution set. A symmetry group $G$ of the differential equations $\Delta=0$ is a group $G$ which acts on the solution space $\mathcal{S}$.

Two fundamental problems with regards to symmetry are;

Lie 1. Determine a non-trivial symmetry group $G$ for the differential equations $\Delta=0$ without explicit knowledge of the space of solutions. 
Lie 2. Given a symmetry group $G$ of $\Delta=0$ use this to find explicit solutions to $\Delta=0$.

The books (Olver, 1999; Bluman and Kumei, 1989; Osvianikov, 1982 ) provide a partial solution to Lie 1 . We won't say too much about this problem but take the position here that for the differential equations $\Delta=0$ a symmetry group $G$ is given.

Given $s \in \mathcal{S}$ the isotropy subgroup $G_{s} \subset G$ is the symmetry group of the solution $s$. For a given $G$ the set of fixed points $\mathcal{S}^{G}$, or solutions with $G_{s}=G$, are called $G$-invariant solutions.

Example 2.1. Let $\Delta=u_{x x}+u_{y y}=0$ be Laplace's equation for $u \in$ $C^{2}\left(\mathbb{R}^{2}-0, \mathbb{R}\right)$ the set of $C^{2}$ real-valued functions on $\mathbb{R}^{2}-0$, and denote by $\mathcal{S} \subset C^{2}\left(\mathbb{R}^{2}-0, \mathbb{R}\right)$ the subset of solutions. Let $G=S O(2)$ act on $\mathbb{R}^{2}-0$ in the standard way. If $f(\mathbf{x}) \in C^{2}\left(\mathbb{R}^{0}-0, \mathbb{R}\right)$ and $A \in G$ then $\mu(A, f)(\mathbf{x})=f\left(A^{-1} \mathbf{x}\right), \mathbf{x} \in \mathbb{R}^{2}-0$ is an action of $G$ on $C^{2}\left(\mathbb{R}^{2}-0, \mathbb{R}\right)$. It is a simple matter to check that if $f(\mathbf{x})$ is a solution to Laplace's equation then $\mu(A, f)$ is also a solution, so $S O(2)$ is a symmetry group of Laplace's equation.

We now find $\mathcal{S}^{G}$, the $S O(2)$ invariant solutions. To find $\mathcal{S}^{G}$ we note that $\mathcal{S}^{G}=\mathcal{S} \cap C^{2}\left(\mathbb{R}^{2}-0, \mathbb{R}\right)^{G}$. The set $C^{2}\left(\mathbb{R}^{2}-0, \mathbb{R}\right)^{G}$ consists of functions fixed by the action so $f \in C^{2}\left(\mathbb{R}^{2}-0, \mathbb{R}\right)^{G}$ if and only if

$$
\mu(A, f)(\mathbf{x})=f\left(A^{-1} \mathbf{x}\right)=f(\mathbf{x}), \quad \text { for all } A \in S O(2), \quad \mathbf{x} \in \mathbb{R}^{2}-0 .
$$

This implies $f(\mathbf{x})$ is constant on the orbits of $S O(2)$ acting on $\mathbb{R}^{2}-$ 0 , and so $f(\mathbf{x})=f(r)$ where $r=\sqrt{x^{2}+y^{2}}$. For $f \in \mathcal{S} \cap C^{2}\left(\mathbb{R}^{2}-\right.$ $0, \mathbb{R})^{G}$ we require $f(\mathbf{x})=f(r)$ to satisfy Laplace's equation which, upon substitution, yields

$$
f^{\prime \prime}+\frac{1}{r} f^{\prime}=0
$$

and so $f(r)=c_{0} \log r+c_{1}$. These are the $S O(2)$-invariant solutions to Laplace's equation.

Most explicit solutions to differential equations like the Schwarzshild solution to the Einstein equations, the Veronese solution to the harmonic map equations, and the fundamental solutions to scalar partial differential equations are group invariant solutions. (Anderson et. al., 2000).

At the other extreme lie the solutions which have no symmetry.

REMARK 2.1. The focus of this article is to give a mechanism which uses the symmetry of a differential equation to produce solutions which have no symmetry. 
We approach this problem using exterior differential systems.

In the next section we show how to replace the differential equations $\Delta=0$ with an exterior differential system $\mathcal{I}$. In section 4 we define the reduced exterior differential system $\overline{\mathcal{I}}=0$ whose integral manifolds will allow us to construct solutions to $\Delta=0$ without symmetry. Section 4 also contains several illustrative examples of this reduction procedure. In section 5 we consider Pfaffian EDS and answer the basic question of when the reduction of a Pfaffian EDS is also a Pfaffian EDS. Section 6 shows how to reproduce solutions to the original system $\mathcal{I}$ from integral manifolds of the reduced system $\overline{\mathcal{I}}$. This is known as the reconstruction problem for EDS with symmetry. The integral manifolds produced this way are the sought after solutions which have no symmetry. The detailed proofs of the theorems are given in section 7 .

\section{Exterior Differential Systems with Symmetry}

Let $M$ be an $n$-dimensional differentiable manifold, and let $\Omega^{*}(M)$ be the exterior algebra of all differential forms on $M$. An exterior differential system (EDS) on $M$ is a differential ideal $\mathcal{I}$ in the exterior algebra $\Omega^{*}(M)$ of differential forms on $M$. The ideal $\mathcal{I}$ is a direct sum of its homogeneous components $\mathcal{I}^{k}=\mathcal{I} \cap \Omega^{k}(M)$,

$$
\mathcal{I}=\oplus_{k \geq 0} \mathcal{I}^{k}
$$

It is customary to assume $\mathcal{I}^{0}=0$, so that $\mathcal{I}$ contains no smooth functions. That $\mathcal{I}$ is closed under differentiation is equivalent to the statement that if $\omega \in \mathcal{I}^{k}$, then $d \omega \in \mathcal{I}^{k+1}$.

We also suppose that $\mathcal{I}$ is of constant rank in the following sense. For each $k=1, \ldots n$, we assume that there are sub-bundles $I^{k} \subset \Lambda^{k}\left(T^{*} M\right)$ of rank $r_{k}$ such that $\mathcal{I}^{k}$ is the space of sections of $I^{k}$.

An integral manifold of an EDS $\mathcal{I}$ is an immersion $s: N \rightarrow M$ such that $s^{*} \theta=0$, for all $\theta \in \mathcal{I}$.

Let $S$ be a subset of $\Omega^{*}(M)$. The algebraic ideal generated by $S$ is denoted by $\langle S\rangle$ so that the exterior differential system generated by $S$ is $\langle S, d S\rangle$. The notation mod $S$ means computed modulo the ideal $\langle S\rangle$ in the appropriate setting. For example $\mathcal{I}^{2} \bmod \mathcal{I}^{1}$ is precisely $\mathcal{I}^{2} \bmod \left(<\mathcal{I}^{1}>\cap \Omega^{2}(M)\right)$, but this more cumbersome notation will not be used. The standard reference on EDS is $\left(\mathrm{BCG}^{3}, 1991\right)$.

If $U$ is an open subset of $M$ we write $\Omega^{*}(U), \mathcal{I}(U), T^{*} U, I^{k}(U)$, and so on to denote the restriction to $U$. Let $U$ be an open set in $M$ we say the set of forms $\left\{\kappa^{w_{k}}\right\}$ form a local basis for $\mathcal{I}^{k}(U) \bmod \mathcal{I}^{k-1}(U)$ if $\kappa^{w_{k}}$ are point-wise linearly independent and every $\kappa \in \mathcal{I}^{k}(U)$ can be 
written as

$$
\kappa=\rho_{w_{k}} \kappa^{w_{k}}+\rho
$$

where $\rho_{w_{k}} \in C^{\infty}(U), \rho \in \mathcal{I}^{k-1}(U)$, and $\rho_{w_{k}}$ are unique.

Given an $\operatorname{EDS} \mathcal{I}$, a local basis $\mathcal{B}(U)$ on the open set $U \subset M$ is a subset $\mathcal{B}(U) \subset \mathcal{I}(U)$ given by

$$
\mathcal{B}(U)=\left\{\theta^{w_{1}}, \alpha^{w_{2}}, \ldots, \kappa^{w_{k}}, \ldots, \nu^{w_{n}}\right\}
$$

where $\left\{\theta^{w_{1}}\right\}$ are a basis for $\mathcal{I}^{1}(U),\left\{\alpha^{w_{2}}\right\}$ are a basis for $\mathcal{I}^{2}(U) \bmod \mathcal{I}^{1}(U)$, $\ldots,\left\{\nu^{w_{n}}\right\}$ are a basis for $\mathcal{I}^{n}(U) \bmod \mathcal{I}^{n-1}(U)$. If $\mathcal{B}(U)$ is a local basis then given $\omega \in \mathcal{I}^{k}(U)$ there exists unique $\rho_{w_{l}} \in \Omega^{k-l}(U)$ such that

$$
\omega=\rho_{w_{1}} \wedge \theta^{w_{1}}+\ldots \rho_{w_{k}} \wedge \kappa^{w_{k}} .
$$

If $\mathcal{I}$ is a constant rank EDS system, it is easy to check that about each $x \in M$ there an open neighbourhood $U$ of $x$ and local basis $\mathcal{B}(U)$ for $\mathcal{I}(U)$. It is also easy to check that if $\mathcal{B}(U)$ is a local basis for $\mathcal{I}$ on $U$ then $s: N \rightarrow U$ is an integral manifold if and only if $s^{*} \mathcal{B}=0$.

Most exterior differential systems that arise in practice are generated algebraically by forms of low degree (in fact usually of degree one or two) and sometimes this may be used to simplify the determination of $\overline{\mathcal{I}}$. We make this formal in the next definition.

DEFINITION 3.1. An EDS $\mathcal{I}$ is generated algebraically by its first $k$-homogeneous components if for each $x \in M$ there exits an open neighborhood $U$ of $x$ such that

$$
\mathcal{I}(U)=<\mathcal{I}^{1}(U), \mathcal{I}^{2}(U), \ldots, \mathcal{I}^{k}(U)>.
$$

If $\mathcal{I}$ is algebraically generated by its first $k$-homogeneous components, then $s: N \rightarrow M$ is an integral manifold if and only if $s^{*} \theta=0$ for all $\theta \in \mathcal{I}^{l}, l=1, \ldots, k$. It is easy to see if $\mathcal{I}$ is also of constant rank then there exists a local basis $\mathcal{B}(U)$ consisting of forms in $\mathcal{I}^{l}(U)$ for $1 \leq l \leq k$.

We now turn to the relationship between differential equations and EDS. Every sufficiently regular system of differential equations $\Delta=0$ for $m$ real-valued functions of $n$ real variables can be encoded, at least locally, as an EDS in the following standard way. Let $J^{k}\left(\mathbb{R}^{n}, \mathbb{R}^{m}\right)$ denote the jet space of functions from $\mathbb{R}^{n}$ to $\mathbb{R}^{m}$. The standard coordinates on $J^{k}\left(\mathbb{R}^{n}, \mathbb{R}^{m}\right)$ are

$$
\left(x^{i}, u^{a}, u_{i_{1}}^{a}, u_{i_{1} i_{2}}^{a}, \ldots, u_{i_{1} i_{2} \ldots i_{k}}^{a}\right), \quad 1 \leq i, i_{1}, i_{2}, \ldots i_{k} \leq n, 1 \leq a \leq m .
$$


The contact one forms on $J^{k}\left(\mathbb{R}^{n}, \mathbb{R}^{m}\right)$ are given for $l=0, \ldots, k-1$ by $\theta_{i_{1} \ldots i_{l}}^{a}=d u_{i_{1} \ldots i_{l}}^{a}-u_{i_{1} \ldots i_{l} i_{l+1}}^{a} d x^{i_{l+1}}, 1 \leq i, i_{1}, i_{2}, \ldots, i_{l+1} \leq n, 1 \leq a \leq m$.

These forms generate the contact ideal $\mathcal{C}$ which is the differential ideal

$$
\mathcal{C}=<\theta^{a}, \theta_{i_{1}}^{a}, \ldots, \theta_{i_{1} \ldots i_{k-1}}^{a}, d \theta^{a}, d \theta_{i_{1}}^{a}, \ldots, d \theta_{i_{1} \ldots i_{k-1}}^{a}>.
$$

A system of differential equations is the zero set of a smooth function $\Delta: J^{k}\left(\mathbb{R}^{n}, \mathbb{R}^{m}\right) \rightarrow \mathbb{R}^{p}$. Subject to rank conditions on the Jacobian of $\Delta$ this zero-set defines the embedded submanifold

$$
M=\left\{\left(x^{i}, u^{a}, \ldots, u_{i_{1} \ldots i_{k}}^{a}\right) \mid \Delta=0\right\} .
$$

The restriction (or pullback by the inclusion map) of the contact ideal to $M$ defines an EDS $\mathcal{I}$ on $M$. A solution to the differential equations $\Delta=0$ defines an integral manifold $s: N \rightarrow M$ of $\mathcal{I}$ such that $s^{*}\left(d x^{1} \wedge\right.$ $\left.d x^{2} \ldots \wedge d x^{n}\right) \neq 0$. Conversely every integral manifold $: N \rightarrow M$ such that $s^{*}\left(d x^{1} \wedge d x^{2} \ldots \wedge d x^{n}\right) \neq 0$ gives rise to a solution of $\Delta=0$.

Example 3.1. The $F$-Gordon equation $u_{x y}=F\left(x, y, u, u_{x}, u_{y}\right)$ defines the 7 dimensional manifold $M$ with coordinates $\left(x, y, u, u_{x}, u_{y}, u_{x x}, u_{y y}\right)$ as a submanifold of $J^{2}\left(\mathbb{R}^{2}, \mathbb{R}\right)$ by

$$
\left(x, y, u, u_{x}, u_{y}, u_{x x}, u_{y y}\right) \rightarrow\left(x, y, u, u_{x}, u_{y}, u_{x x}, u_{x y}=F, u_{y y}\right) .
$$

The restriction (or pullback by inclusion) of the contact ideal produces the EDS on $M$ given by

$$
\begin{aligned}
& \mathcal{I}=<d u-u_{x} d x-u_{y} d y, \quad d u_{x}-u_{x x} d x-F d y, \quad d u_{y}-F d x-u_{y y} d y \\
& d x \wedge d u_{x x}+d y \wedge d F, d x \wedge d F+d y \wedge d u_{y y}>\text {. }
\end{aligned}
$$

If $u=f(x, y)$ is a $C^{\infty}$ solution to the $F$-Gordon equation $u_{x y}=F$, then the map $s: \mathbb{R}^{2} \rightarrow M$ defined by

$$
s(x, y)=\left(x, y, u=f, u_{x}=\partial_{x} f, u_{y}=\partial_{y} f, u_{x x}=\partial_{x x}^{2} f, u_{y y}=\partial_{y}^{2} f\right)
$$

is an integral manifold of $\mathcal{I}$. Conversely, if

$$
s(x, y)=\left(x, y, u=f_{1}, u_{x}=f_{2}, u_{y}=f_{3}, u_{x x}=f_{4}, u_{y y}=f_{5}\right)
$$

where the $f_{i}$ are functions of $(x, y)$, is an integral manifold of $\mathcal{I}$, then one readily finds that

$$
f_{2}=\partial_{x} f_{1}, f_{3}=\partial_{y} f_{1}, f_{4}=\partial_{x}^{2} f_{1}, f_{5}=\partial_{y}^{2} f_{1}
$$

and $u=f_{1}(x, y)$ satisfies the $F$-Gordon equation $u_{x y}=F$. In this way the EDS $\mathcal{I}$ encodes the $F$-Gordon equation. 
The next example shows that a differential equation $\Delta=0$ can be encoded as an EDS in different ways.

Example 3.2. On $M=\mathbb{R}^{5}$ with coordinates $\left(x, y, u, u_{x}, u_{y}\right)$ let

$$
\begin{gathered}
\mathcal{I}=<d u-u_{x} d x-u_{y} d y, d x \wedge d u_{x}+d y \wedge d u_{y}, \\
\left(d u_{x}-F\left(x, y, u, u_{x}, u_{y}\right) d y\right) \wedge d x>.
\end{gathered}
$$

If $u=f(x, y)$ is a solution to $u_{x y}=F\left(x, y, u, u_{x}, u_{y}\right)$ then the map $s: \mathbb{R}^{2} \rightarrow M$,

$$
s(x, y)=\left(x, y, u=f, u_{x}=\partial_{x} f, u_{y}=\partial_{y} f\right)
$$

is an integral manifold to $\mathcal{I}$. Conversely, if

$$
s(x, y)=\left(x, y, u=f(x, y), u_{x}=g(x, y), u_{y}=h(x, y)\right)
$$

is an integral manifold of $\mathcal{I}$, then it is easy to check that

$$
g=\partial_{x} f, h=\partial_{y} f
$$

and $u=f(x, y)$ satisfies the $F$-Gordon equation $u_{x y}=F$. This EDS also encodes the $F$-Gordon equation.

A classical contact symmetry of the differential equations $\Delta=0$ is a diffeomorphism $\Phi: J^{k}\left(\mathbb{R}^{m}, \mathbb{R}^{n}\right) \rightarrow J^{k}\left(\mathbb{R}^{m}, \mathbb{R}^{n}\right)$ such that $\Phi^{*} \mathcal{C}=$ $\mathcal{C}$ and $\Phi(M)=M$. Each classical contact symmetry is a symmetry of the differential equations $\Delta=0$ (Olver, 1999). A classical contact symmetry restricts to a diffeomorphism $\Phi: M \rightarrow M$ such that $\Phi^{*} \mathcal{I}=\mathcal{I}$ and this leads to the following general definition.

DEFINITION 3.2. A symmetry of the EDS $\mathcal{I}$ on a manifold $M$ is a diffeomorphism $\phi: M \rightarrow M$ satisfying $\phi^{*} \mathcal{I}=\mathcal{I}$. A symmetry group of $\mathcal{I}$ is a subgroup $G \subset \operatorname{Diff}(M)$ whose elements are symmetries of $\mathcal{I}$.

If $\phi$ a symmetry of the EDS $\mathcal{I}$ then

$$
\phi^{*} \mathcal{I}^{k}=\mathcal{I}^{k} \quad \text { and } \quad \phi^{*} I^{k}=I^{k} .
$$

See (Anderson et. al., 1993) for more information on the relationship between symmetries of EDS associated to differential equations and classical contact symmetries.

LEMMA 3.1. If $\phi$ is a symmetry of the EDS $\mathcal{I}$ and $s: N \rightarrow M$ is an integral manifold of $\mathcal{I}$ then $\phi \circ s$ is an integral manifold of $\mathcal{I}$. 
Proof. We compute $(\phi \circ s)^{*} \mathcal{I}=s^{*} \phi^{*} \mathcal{I}=s^{*} \mathcal{I}=0$, and simply note that $\phi \circ s$ is an immersion.

This lemma just says that symmetries of an EDS map integral manifolds to integral manifolds.

\section{Reduction of Exterior Differential Systems - Introduction and Examples}

In this section we define the reduction of an exterior differential system by a symmetry group $G$. We find that if the action satisfies a certain transversality condition then the reduction of an EDS can readily be computed. Four examples are then given in detail.

The definition of a symmetry group $G$ of an EDS allows $G$ to be an arbitrary subgroup of $\operatorname{Diff}(M)$. We will restrict our attention to symmetry groups which are Lie groups and where the action $\mu: G \times$ $M \rightarrow M$ is smooth, even though most of the concepts presented don't require this restriction. We will also assume, without loss of generality, that all actions are effective.

The action of the Lie group $G$ on the manifold $M$ is regular (Olver, 1999 ) if the quotient space $M / G$, which we now denote by $\bar{M}$, is a differentiable manifold with the projection map q : $M \rightarrow M / G$ being a submersion. If $G$ acts regularly on $M$ with $q$-dimensional orbits, then $\bar{M}=M / G$ is an $n-q$ dimensional manifold.

Let $\Gamma$ be the Lie algebra of infinitesimal generators of the action of $G$ (Olver, 1999), and let $\boldsymbol{\Gamma} \subset T M$ denote the corresponding completely integrable distribution with $\boldsymbol{\Gamma}_{x}=\operatorname{span}\left\{\Gamma_{x}\right\}$. We use the notation $X \in$ $\boldsymbol{\Gamma}$ to indicate that $X$ is vector field with values in $\boldsymbol{\Gamma}$, while $X \in \boldsymbol{\Gamma}_{x}$ denotes $X$ is a tangent vector at $x$ taking values in $\boldsymbol{\Gamma}_{x}$.

We now give the definition for the reduction of an EDS, (Itskov, 2001).

DEFINITION 4.1. Let $G$ be a Lie group which acts regularly on the manifold $M$ and is a symmetry group of the $E D S \mathcal{I}$ on $M$. The reduced $E D S \overline{\mathcal{I}} \subset \Omega^{*}(\bar{M})$ is defined by

$$
\overline{\mathcal{I}}=\left\{\bar{\alpha} \in \Omega^{*}(\bar{M}) \mid \mathbf{q}^{*} \bar{\alpha} \in \mathcal{I}\right\} .
$$

The reduced EDS $\overline{\mathcal{I}}$ is clearly a differential ideal whose homogeneous components are

$$
\overline{\mathcal{I}}^{k}=\left\{\bar{\alpha} \in \Omega^{k}(\bar{M}) \mid \mathbf{q}^{*} \bar{\alpha} \in \mathcal{I}^{k}\right\} .
$$

While the definition of the reduced EDS $\overline{\mathcal{I}}$ is fairly simple, the actual determination of the properties of $\overline{\mathcal{I}}$ is not. For example if $\mathcal{I}$ satisfies 
the constant rank hypothesis there is no reason to believe that $\overline{\mathcal{I}}$ will be of constant rank.

In order to compute $\overline{\mathcal{I}}$ and study its properties we introduce the subalgebra of semi-basic differential forms $\mathcal{A}_{s b}$ in $\mathcal{I}$ defined by

$$
\mathcal{A}_{s b}=\left\{\alpha \in \mathcal{I} \mid \iota_{X} \alpha=0, \quad \text { for all } X \in \Gamma\right\} .
$$

The algebra $\mathcal{A}_{s b}$ is a direct sum of its homogeneous components $\mathcal{A}_{s b}^{k}$ given by

$$
\mathcal{A}_{s b}^{k}=\left\{\alpha \in \mathcal{I}^{k} \mid \iota_{X} \alpha=0, \quad \text { for all } X \in \Gamma\right\}=\mathcal{I}^{k} \cap \Omega_{s b}^{k}(M),
$$

where $\Omega_{s b}^{*}(M)$ is defined in Appendix A. Algebraically $\mathcal{A}_{s b} \subset \Omega_{s b}^{*}(M)$ is an ideal, and so a local basis $\mathcal{B}_{s b}(U)$ for the algebra $\mathcal{A}_{s b}$ satisfies the same conditions in (2) except that $\rho_{w_{l}} \in \Omega_{s b}^{k-l}(U)$.

In a similar manner to $\mathcal{A}_{s b}$ we can define the subset $A_{s b}^{k} \subset I^{k}$ of semi-basic $k$-forms which is given point-wise by

$$
A_{s b, x}^{k}=\left\{\alpha \in I_{x}^{k} \mid \iota_{X} \alpha=0, \quad \text { for all } X \in \Gamma_{x}\right\}=I_{x}^{k} \cap \Lambda_{s b, x}^{k}(M) .
$$

It is easy to check that if $A_{s b}^{k}$ is a bundle, then $\mathcal{A}_{s b}^{k}$ are the sections of this bundle.

REMARK 4.1. Most geometric properties of $\overline{\mathcal{I}}$ can inferred from the properties of $\mathcal{A}_{s b}$ and the sets $A_{s b}^{k}$.

An illustration of Remark 4.1 is given by Lemma 7.1 where we prove that if $A_{s b}^{k}$ is a bundle then $\bar{I}^{k}$ is a bundle and consequently, if this is true for all $k=1, \ldots, n$, then $\overline{\mathcal{I}}$ is of constant rank.

We now look at some other useful relationships between $\mathcal{A}_{s b}$ and $\overline{\mathcal{I}}$ that will assist in computing $\overline{\mathcal{I}}$. The first point we note is that if $\bar{\alpha} \in \overline{\mathcal{I}}$ then $\mathbf{q}^{*} \bar{\alpha} \in \mathcal{A}_{s b}$. More precisely by Lemma A.3 or (20), we have

$$
\mathcal{A}_{s b}^{G}=\mathbf{q}^{*}(\overline{\mathcal{I}}) .
$$

The local relationship between $\mathcal{A}_{s b}$ and $\overline{\mathcal{I}}$ that is given in the next lemma and its corollary is extremely useful.

LEMMA 4.1. Let $U$ be an open set of $M$ admitting a cross-section $\sigma: \bar{U} \rightarrow U$, where $\bar{U}=\mathbf{q}(U)$. If $\alpha \in \mathcal{A}_{s b}^{k}(U)$ then $\sigma^{*} \alpha \in \overline{\mathcal{I}}^{k}(U)$. Therefore $\sigma^{*}\left(\mathcal{A}_{s b}^{k}(U)\right)=\overline{\mathcal{I}}^{k}(\bar{U})$.

Proof. By Lemma A.2 we have $\mathbf{q}^{*} \sigma^{*} \alpha_{\sigma(\bar{x})} \in I_{\sigma(\bar{x})}^{k}$ for all $\bar{x} \in \bar{U}$. However both $\mathbf{q}^{*} \sigma^{*} \alpha$ and $I^{k}$ are invariant and so $\mathbf{q}^{*} \sigma^{*} \alpha \in \mathcal{I}^{k}(U)$ which implies $\sigma^{*} \alpha \in \overline{\mathcal{I}}^{k}(\bar{U})$. The fact that $\sigma^{*}$ is onto follows from $\sigma^{*} \mathbf{q}^{*} \bar{\alpha}=\bar{\alpha}$ for any $\bar{\alpha} \in \overline{\mathcal{I}}^{k}(\bar{U})$.

From this we have the following corollary. 
COROLLARY 4.1. Let $U$ be an open set, $\sigma: \bar{U} \rightarrow U$ a cross-section where $\bar{U}=\mathbf{q}(U)$, and $\mathcal{B}_{s b}(U)$ a local basis for $\mathcal{A}_{s b}(U)$. The pullback $\sigma^{*} \mathcal{B}_{s b}(U)$ is a local basis for $\overline{\mathcal{I}}(\bar{U})$.

Without additional hypothesis about the relationship between the symmetry group $G$ and $\mathcal{I}$ it is difficult to make inferences about $\overline{\mathcal{I}}$. We now introduce a geometric condition on the action of $G$ which, if satisfied, allows us to precisely determine the structure of $\overline{\mathcal{I}}$. In order to give this definition we use the notation that if $J \subset T^{*} M$ is a sub-bundle then $J^{\perp} \subset T M$ is the annihilator given point-wise by

$$
J_{x}^{\perp}=\left\{Y \in T_{x} M \mid \iota_{Y} \alpha=0 \text { for all } \alpha \in J_{x}\right\} .
$$

DEFINITION 4.2. Let $\mathcal{I}$ be an exterior differential system and $G$ a symmetry group of $\mathcal{I}$ which acts regularly on $M$. The action of $G$ is transverse to $\mathcal{I}^{1}$ if $\boldsymbol{\Gamma} \cap\left(I^{1}\right)^{\perp}=0$.

Two useful properties of transverse actions are given in the next proposition.

PROPOSITION 4.1. Let $G$ be a symmetry group of the EDS $\mathcal{I}$ which acts transversally to $\mathcal{I}^{1}$. If $s: N \rightarrow M$ is an integral manifold of $\mathcal{I}$ the symmetry group of $s$ is discrete and $\mathbf{q} \circ s$ is an integral manifold of $\overline{\mathcal{I}}$.

Proof. If $s: N \rightarrow M$ is an integral manifold then $s_{*}: T_{x} N \rightarrow\left(I^{1}\right)^{\perp}$ but transversality implies $s_{*}\left(T_{x} N\right) \cap \boldsymbol{\Gamma}=0$. By the effectiveness of the action of $G$ this implies that $s$ is not invariant with respect to any one parameter subgroup of $G$. Therefore the symmetries of $s$ are discrete.

For the second part of the proposition let $\bar{\alpha} \in \overline{\mathcal{I}}$. Then $\mathbf{q}^{*} \bar{\alpha} \in \mathcal{I}$ and $(\mathbf{q} \circ s)^{*} \bar{\alpha}=s^{*} \mathbf{q}^{*} \bar{\alpha}=0$ as required. To finish the proof we need to show that $\mathbf{q} \circ s$ is an immersion. Suppose $Y \in T_{x} N$ and that $(\mathbf{q} \circ s)_{*} Y=$ 0 . Now because $s$ is an integral manifold $s_{*} Y \in\left(I_{s(x)}^{1}\right)^{\perp}$, and so, if $(\mathbf{q} \circ s)_{*} Y=0$ then $s_{*} Y \in \operatorname{ker} \mathbf{q}_{*}=\boldsymbol{\Gamma}$ and hence $s_{*} Y \in \boldsymbol{\Gamma} \cap\left(I^{1}\right)^{\perp}$. By hypothesis this implies that $s_{*} Y=0$. But $s$ is an immersion and so $Y=0$.

We now present a proposition which will allow us to compute $\overline{\mathcal{I}}$ under the transversality hypothesis.

PROPOSITION 4.2. Let $\mathcal{I}$ be an EDS that is algebraically generated by its first $k$-homogeneous components and let $G$ be a symmetry group of $\mathcal{I}$ which acts transversally to $\mathcal{I}^{1}$. Then about each point $x \in M$ there exists an open set $U$ and local basis

$$
\mathcal{B}(U)=\left\{\eta^{i}, \theta^{w^{1}}, \alpha^{w_{2}}, \ldots, \kappa^{w_{k}}\right\}
$$


$1 \leq i \leq q, 1 \leq w_{l} \leq \delta_{l}$ for $l=1 \ldots k$, where $\theta^{w_{1}} \in \mathcal{A}_{s b}^{1}(U), \alpha^{w_{2}} \in$ $\mathcal{A}_{s b}^{2}(U), \ldots, \kappa^{k} \in \mathcal{A}_{s b}^{k}(U)$. Moreover given any local basis of the form (5), then

$$
\mathcal{B}_{s b}(U)=\left\{\theta^{w^{1}}, \alpha^{w_{2}}, \ldots, \kappa^{w_{k}}\right\}
$$

is a local basis for $\mathcal{A}_{s b}(U)$.

This proposition in conjunction with Corollary 4.1 allows us to compute $\overline{\mathcal{I}}$ locally by finding a local basis for $\mathcal{A}_{s b}$ consisting of forms of low degree, and pulling this back by a local cross-section to the action of $G$. All of these steps are algebraic which makes determining $\overline{\mathcal{I}}$ algebraic (locally). See Corollary 7.3 for some other consequences of this proposition.

We apply the argument above to the examples where the EDS are generated by their first two homogeneous components. We work on an open set $U$ where the action is transverse to $\mathcal{I}^{1}(U)$ and where there exists a cross-section $\sigma: \bar{U} \rightarrow U$. We then find a local basis for $\mathcal{A}_{s b}(U)$ which by Proposition 4.2 consists of one-forms and two forms (we leave the choice of $\eta^{i}$ to the reader). By Corollary 4.1 these pull-back to give a local basis for $\overline{\mathcal{I}}(\bar{U})$.

Example 4.1. On $M=\left\{\left(x, y, u, u_{x}, u_{y}\right) \in \mathbb{R}^{5} \mid x+y \neq 0\right\}$ let

$\mathcal{I}=<d u-u_{x} d x-u_{y} d y, \quad d u_{x} \wedge d x+d u_{y} \wedge d y, \quad\left(d u_{x}-\frac{\sqrt{u_{x} u_{y}}}{x+y} d y\right) \wedge d x>$.

By definition, $\mathcal{I}$ is algebraically generated by its first two homogeneous components, and $\mathcal{I}$ encodes the differential equation of $F$-Gordon type (see Example 3.2),

$$
u_{x y}=\frac{\sqrt{u_{x} u_{y}}}{x+y} .
$$

We reduce $\mathcal{I}$ by the translational symmetry $u \rightarrow u+c, c \in G=\mathbb{R}$. The Lie algebra of infinitesimal generators on $M$ is $\Gamma=\operatorname{span}\left\{\partial_{u}\right\}$. The transversality condition $\Gamma \cap\left(I^{1}\right)^{\perp}=0$ is satisfied everywhere on $M$. Note that there are no $G$-invariant solutions to (6).

Let $(x, y, v, w)$ be coordinates on the quotient $\bar{M}=M / G=\mathbb{R}^{4}$ and let $\sigma$ be the map

$$
\sigma(x, y, v, w)=\left(x=x, y=y, u=0, u_{x}=v, u_{y}=w\right)
$$

which is easily checked to be transverse to the orbits of $G$ on $M$, and so is a local cross-section. We now find generators for $\overline{\mathcal{I}}$ simply by finding a basis for $\mathcal{A}_{s b}^{1}$ and $\mathcal{A}_{s b}^{2} \bmod \mathcal{A}_{s b}^{1}$ and pulling them back with $\sigma$. The forms are found to be

$\mathcal{A}_{s b}^{1}=\{0\}, \mathcal{A}_{s b}^{2}=\operatorname{span}\left\{d x \wedge d u_{x}+d y \wedge d u_{y},\left(d u_{x}-\frac{\sqrt{u_{x} u_{y}}}{x+y} d y\right) \wedge d x\right\}$. 
Using the cross-section $\sigma$ and pulling back the basis gives on account of Corollary 4.1,

$$
\overline{\mathcal{I}}=<d x \wedge d v+d y \wedge d w, \quad\left(d v-\frac{\sqrt{v w}}{x+y} d y\right) \wedge d x>.
$$

This is the $s=0$ hyperbolic system (Bryant et. al., 1995) which encodes the differential equations

$$
v_{y}=\frac{\sqrt{v w}}{x+y}, \quad w_{x}=\frac{\sqrt{v w}}{x+y} .
$$

It is interesting to note that both the original scalar equation (6) and the system (7) in this example are integrable by the method of Darboux. Moreover equations (7) are linearizable (Bryant et. al., 1995) while the original equation is not. In a forthcoming paper we will consider the reduction of Darboux integrable equations in more detail.

Example 4.2. We start with the EDS

$\mathcal{I}=<d u-u_{x x} d t-u_{x} d x, \quad d t \wedge d u_{x x}+d x \wedge d u_{x}, \quad\left(d u_{x}-u_{x x} d x\right) \wedge d t>$

on the five manifold $M=\mathbb{R}^{5}$ with coordinates $\left(t, x, u, u_{x}, u_{x x}\right)$. This EDS encodes the heat equation $u_{t}=u_{x x}$ and $\mathcal{I}$ is algebraically generated by its first two homogeneous components. The action of $G=\mathbb{R}^{*}$ on $M$ defined by

$$
\mu\left(\lambda, t, x, u, u_{x}, u_{x x}\right)=\left(t, x, \lambda u, \lambda u_{x}, \lambda u_{x x}\right), \lambda \in G
$$

is easily checked to be a symmetry group of $\mathcal{I}$. The Lie algebra of infinitesimal generators on $M$ is $\Gamma=\operatorname{span}\left\{u \partial_{u}+u_{x} \partial_{u_{x}}+u_{x x} \partial_{u_{x x}}\right\}$. We find that the transversality condition $\Gamma \cap\left(I^{1}\right)^{\perp}=0$ is satisfied only at points of $M$ where $u \neq 0$. So we work on the open set $U=M-\{u=0\}$ where the action is transverse. Note that working on the set $u \neq 0$ eliminates the scale invariant solution $u=0$.

Let $\left(t, x, w, w_{x}\right)$ be coordinates on the quotient $\bar{U}=U / G=\mathbb{R}^{4}$ and let $\sigma: \bar{U} \rightarrow U$ be the cross-section

$$
\sigma\left(t, x, w, w_{x}\right)=\left(t, x, u=1, u_{x}=w, u_{x x}=w_{x}+w^{2}\right) .
$$

According to the argument above, we need to find a local basis for the semi-basic forms $\mathcal{A}_{s b}^{1}(U)$ and $\mathcal{A}_{s b}^{2}(U)$. The forms are

$$
\begin{aligned}
\mathcal{A}_{s b}^{1}(U)= & \{0\} \\
\mathcal{A}_{s b}^{2}(U)= & \operatorname{span}\left\{\left(-u_{x}^{2}+u u_{x x}\right) d t \wedge d x+u_{x} d t \wedge d u-u d t \wedge d u_{x},\right. \\
& \left.-u_{x x} d t \wedge d u+u d t \wedge d u_{x x}-u_{x} d x \wedge d u+u d x \wedge d u_{x}\right\}
\end{aligned}
$$


By pulling back the given basis forms in $\mathcal{A}_{s b}^{2}(U)$ using $\sigma$ gives

$$
\overline{\mathcal{I}}=<\left(d w-w_{x} d x\right) \wedge d t, \quad d\left(w_{x}+w^{2}\right) \wedge d t+d w \wedge d x>.
$$

This exterior differential system encodes Burgers' equation $w_{t}=w_{x x}+$ $2 w w_{x}$ on a four manifold, see (Bryant and Griffiths, 1995) and (Krasilcshchik and Vinogradov, 1984). In this example it is interesting to note that the heat equation which is in encoded by $\mathcal{I}$ is linear while Burgers' equation which is encoded by $\overline{\mathcal{I}}$ is not. The projection map $\mathbf{q}$ in this example is

$$
\mathbf{q}\left(t, x, u, u_{x}, u_{x x}\right)=\left(t, x, w=u_{x} u^{-1}, w_{x}=\left(u_{x x}-u_{x}^{2}\right) u^{-2}\right) .
$$

By using Proposotion 4.1 we can take any non-vanishing solution to the linear heat equation and map it by $\mathbf{q}$ to a solution to Burgers' equation. This transformation is known as the Hopf-Cole transformation (Bluman and Kumei, 1989).

Example 4.3. The EDS described in section 3 defined by the wave equation $u_{x y}=0$ is

$\mathcal{I}=<d u-u_{x} d x-u_{y} d y, d u_{x}-u_{x x} d x, d u_{y}-u_{y y} d y, d u_{x x} \wedge d x, d u_{y y} \wedge d y>$

on the 7 manifold $M=\mathbb{R}^{7}$ with coordinates $\left(x, y, u, u_{x}, u_{y}, u_{x x}, u_{y y}\right)$. The EDS is algebraically generated by its first two homogeneous components. The group $G=\mathbb{R}^{2}$ acting by translations $x \rightarrow x+a ; y \rightarrow y+b$ is a symmetry group of $\mathcal{I}$. The Lie algebra of infinitesimal generators is $\Gamma=\operatorname{span}\left\{\partial_{x}, \partial_{y}\right\}$ and $\left(I^{1}\right)^{\perp} \cap \boldsymbol{\Gamma}=0$ is satisfied at all points of the open set

$$
U=\left\{\left(x, y, u, u_{x}, u_{y}, u_{x x}, u_{y y}\right) \in \mathbb{R}^{7} \mid u_{x} u_{y} u_{x x} u_{y y} \neq 0\right\} .
$$

A solution to the wave equation $u_{x y}=0$ which is invariant under a connected subgroup of $G$ must be linear and so working on the subset $u_{x} u_{y y} u_{y} u_{x x} \neq 0$ eliminates the invariant solutions.

The transversality condition is satisfied on $U$. Let $\sigma$ be the crosssection $\sigma: \bar{U} \rightarrow U$ given by

$$
\begin{aligned}
& \sigma\left(\alpha, \beta, w, w_{\alpha}, w_{\beta}\right)= \\
& \quad\left(x=0, y=0, u=w, u_{x}=\alpha, u_{y}=\beta, u_{x x}=\frac{\alpha}{w_{\alpha}}, u_{y y}=\frac{\beta}{w_{\beta}}\right)
\end{aligned}
$$

where $\bar{U}=\left\{\left(\alpha, \beta, w, w_{\alpha}, w_{\beta}\right) \in \mathbb{R}^{5} \mid \alpha \beta w_{\alpha} w_{\beta} \neq 0\right\}$. We first compute $\mathcal{A}_{s b}^{1}(U)$ to be

$$
\mathcal{A}_{s b}^{1}(U)=\operatorname{span}\left\{u_{y y} u_{x x} d u-u_{y y} u_{x} d u_{x}-u_{x x} u_{y} d u_{y}\right\}
$$


To compute $\mathcal{A}_{s b}^{2}(U) \bmod \mathcal{A}_{s b}^{1}(U)$ we note that

$$
\begin{aligned}
& d u_{x x} \wedge d x=u_{x x}{ }^{-1} d u_{x} \wedge d u_{x x} \quad \bmod \mathcal{I}^{1}(U) \\
& d u_{y y} \wedge d y=u_{y y}{ }^{-1} d u_{y} \wedge d u_{y y} \quad \bmod \mathcal{I}^{1}(U) .
\end{aligned}
$$

Using these new generators in $\mathcal{I}^{2}(U)$ we find

$$
\mathcal{A}_{s b}^{2}(U)=\operatorname{span}\left\{d u_{x} \wedge d u_{x x}, \quad d u_{y} \wedge d u_{y y}\right\} \bmod \mathcal{A}_{s b}^{1}(U) .
$$

To find $\overline{\mathcal{I}}(\bar{U})$ we pull back by $\sigma$ the basis forms given above to get

$$
\overline{\mathcal{I}}(\bar{U})=<d w-w_{\alpha} d \alpha-w_{\beta} d \beta, \quad d w_{\alpha} \wedge d \alpha, \quad d w_{\beta} \wedge d \beta>
$$

which is the $s=1$ hyperbolic EDS for the wave equation (Bryant et. al., 1995).

Example 4.4. The standard EDS for the system of two partial differential equations in the plane $u_{y}=0, v_{x}=0$ is

$$
\mathcal{I}=<d u-u_{x} d x, \quad d v-v_{y} d y, \quad d x \wedge d u_{x}, \quad d y \wedge d u_{y}>
$$

on the six manifold $M=\mathbb{R}^{6}$ with coordinates $\left(x, y, u, v, u_{x}, v_{y}\right)$. The ideal $\mathcal{I}$ is algebraically generated by its first two homogeneous components. The EDS $\mathcal{I}$ is invariant under $G=\mathbb{R}$ acting by simultaneous translation $u \rightarrow u+a ; v \rightarrow v+a, a \in G$. The Lie algebra of infinitesimal generators is $\Gamma=\operatorname{span}\left\{\partial_{u}+\partial_{v}\right\}$ and $\left(I^{1}\right)^{\perp} \cap \boldsymbol{\Gamma} \neq 0$ so the action is transverse everywhere. Note that there are no $G$-invariant solutions.

Let $\left(x, y, w, w_{x}, w_{y}\right)$ be coordinates on the quotient $\bar{M}=\mathbb{R}^{5}$ and let $\sigma$ be the cross-section

$$
\sigma\left(x, y, w, w_{x}, w_{y}\right)=\left(x, y, w, v=0, w_{x},-w_{y}\right) .
$$

We compute $\overline{\mathcal{I}}$ by first finding

$$
\begin{aligned}
& \mathcal{A}_{s b}^{1}=\operatorname{span}\left\{d u-u_{x} d x-d v+v_{y} d y\right\}, \\
& \mathcal{A}_{s b}^{2}=\operatorname{span}\left\{d x \wedge d u_{x}, d y \wedge d v_{y}\right\}
\end{aligned}
$$

Pulling back using the cross-section $\sigma$ we deduce from that

$$
\overline{\mathcal{I}}=<d w-w_{x} d x-w_{y} d y, \quad d x \wedge d w_{x}, \quad d y \wedge d w_{y}>.
$$

The quotient EDS $\overline{\mathcal{I}}$ encodes the wave equation $u_{x y}=0$ on a five manifold (put $F=0$ in Example 3.2).

\section{Reduction of Pfaffian Systems}

We now apply the previous general discussion to a special but important type of exterior differential system called a Pfaffian system. 
DEFINITION 5.1. An exterior differential system $\mathcal{I}$ is a Pfaffian system if for each point $x \in M$ there exists an open neighbourhood $U \subset M$ such that

$$
\mathcal{I}(U)=\left\langle\theta, d \theta>\quad \text { where } \theta \in \mathcal{I}^{1}(U) .\right.
$$

We will write $I$ instead of $I^{1}$ for Pfaffian systems. The rank of a Pfaffian system $\mathcal{I}$ is $\operatorname{dim} I_{x}$ where $x \in M$ which we will assume is independent of $x$ so that the rank is well defined. Every Pfaffian system is algebraically generated by its first 2 homogeneous components, but the converse is not true.

Examples 4.3 and 4.4 in Section 4 demonstrate that the reduction of a Pfaffian system is not necessarily a Pfaffian system. In Theorem 5.1 below we give sufficient conditions such that the reduction of a Pfaffian system is again a Pfaffian system. The conditions are expressed in terms of the derived system $\mathcal{I}^{\prime}$ of a Pfaffian system.

DEFINITION 5.2. The derived system $\mathcal{I}^{\prime}$ of $\mathcal{I}$ is the Pfaffian system generated by

$$
\mathcal{I}^{\prime}=\left\langle\theta, d \theta>\text { where } \theta \in \mathcal{I}^{1} \text { and } d \theta \equiv 0 \bmod \mathcal{I}^{1} .\right.
$$

The main theorem in this section resolves when the reduction of a Pfaffian system with a symmetry group transverse to $\mathcal{I}^{1}$ is a Pfaffian system.

THEOREM 5.1. Suppose that $G$ is a p-dimensional Lie group acting regularly with q-dimensional orbits on the manifold $M$, and that $G$ is a transverse symmetry group of the rank $r$ Pfaffian system $\mathcal{I}$. The reduced EDS $\overline{\mathcal{I}}$ is a Pfaffian system if and only if $G$ is transverse to $\mathcal{I}^{\prime}$. In this case the rank of $\overline{\mathcal{I}}$ is $r-q$, and $\overline{\mathcal{I}^{\prime}}=(\overline{\mathcal{I}})^{\prime}$

Theorem 5.1 is proved in Section 7. We consider the conditions in Theorem 5.1 by looking at two examples.

Example 5.1. The Pfaffian system in Example 4.3 is transverse to $\mathcal{I}^{1}$ on the set $U$. The derived system is

$$
\mathcal{I}^{\prime}=<d u-u_{x} d x-u_{y} d y, \quad d x \wedge d u_{x}+d y \wedge d u_{y}>
$$

so that $\left(I^{\prime}\right)^{\perp} \cap \boldsymbol{\Gamma}=\left\{u_{y} \partial_{x}-u_{x} \partial_{y}\right\}$ and therefore, according to Theorem $5.1, \overline{\mathcal{I}}$ is not a Pfaffian system. This is verified by the computations in Example 4.3. In particular note that the two-form $d w_{\alpha} \wedge d \alpha \in \overline{\mathcal{I}}^{2}(U)$ but

$$
d w_{\alpha} \wedge d \alpha \notin<\overline{\mathcal{I}}^{1}(U), d \overline{\mathcal{I}}^{1}(U)>.
$$


Example 5.2. In Example 4.4 the action is transverse to $\mathcal{I}^{1}$. The derived system is $\mathcal{I}^{\prime}=\{0\}$ and $\left(I^{\prime}\right)^{\perp} \cap \boldsymbol{\Gamma}=\boldsymbol{\Gamma}$ so $G$ is not transverse to $\left(\mathcal{I}^{\prime}\right)^{1}$. However let's consider the prolongation $\left(\mathrm{BCG}^{3}, 1991\right)$ of $\mathcal{I}$ which consists of writing the EDS for the equations

$$
u_{y}=0, v_{x}=0, u_{x y}=0, u_{y y}=0, v_{x x}=0, v_{x y}=0
$$

which are obtained by including the first derivatives of the equations $u_{y}=0, v_{x}=0$. This gives rise to the Pfaffian system

$$
\begin{aligned}
& \mathcal{J}=<d u-u_{x} d x, \quad d v-v_{y} d y, \quad d u_{x}-u_{x x} d x, \\
& d v_{y}-v_{y y} d y, \quad d x \wedge d u_{x x}, \quad d y \wedge d v_{y y}>
\end{aligned}
$$

on an eight manifold with coordinates $\left(x, y, u, v, u_{x}, v_{y}, u_{x x}, v_{y y}\right)$. The derived system is

$$
\mathcal{J}^{\prime}=<d u-u_{x} d x, \quad d v-v_{y} d y, \quad d x \wedge d u_{x}, \quad d y \wedge d u_{y}>
$$

and $\mathcal{J}^{\prime}=\mathcal{I}$. Therefore $\left(J^{\prime}\right)^{\perp} \cap \boldsymbol{\Gamma}=I^{\perp} \cap \boldsymbol{\Gamma}=0$. By Theorem 5.1, the reduction of $\mathcal{J}$ will be a Pfaffian system. Let $\left(x, y, w, w_{x}, w_{y}, w_{x x}, w_{y y}\right)$ be coordinates on $\bar{M}$ and by pulling back with the cross-section

$$
\begin{aligned}
& \sigma\left(x, y, w, w_{x}, w_{y}, w_{x x}, w_{y y}\right)= \\
& \quad\left(x, y, u=w, v=0, u_{x}=w_{y}, v_{y}=-w_{y}, u_{x x}=w_{x x}, v_{y y}=-w_{y y}\right)
\end{aligned}
$$

we get the Pfaffian system

$$
\begin{gathered}
\overline{\mathcal{J}}=<d w-w_{x} d x-w_{y} d y, d w_{x}-w_{x x} d x, d w_{y}-w_{y y} d y, \\
d x \wedge d w_{x x}, d y \wedge d w_{y y}>.
\end{gathered}
$$

The EDS $\overline{\mathcal{J}}$ encodes the wave equation $w_{x y}=0$ on a 7 manifold in the standard way. For more information on the relationship between prolongation and reduction see (Anderson and Fels, 2004).

REMARK 5.1. A Pfaffian system $\mathcal{I}$ is completely integrable if it satisfies the Frobenius condition $\mathcal{I}^{\prime}=\mathcal{I}$. Under fairly mild conditions on the action of the symmetry group, it is always the case that the reduction of a completely integrable Pfaffian system by a regularly acting symmetry group is again a completely integrable Pfaffian system, (Fels, 2004). 


\section{Reconstruction}

In Lemma 4.1 it was shown that if $\mathcal{I}$ is an EDS, $G$ a symmetry group transverse to $\mathcal{I}^{1}$ and $s: N \rightarrow M$ is an integral manifold of $\mathcal{I}$ then $\mathbf{q} \circ s$ is an integral manifold of $\overline{\mathcal{I}}$. The reconstruction problem is essentially an inverse to this in the following sense: Suppose $\bar{s}: N \rightarrow \bar{M}$ is an integral manifold of $\overline{\mathcal{I}}$, find an integral manifold $s: N \rightarrow M$ of $\mathcal{I}$ such that $\bar{s}=\mathbf{q} \circ s$. Note that if $s$ solves the reconstruction problem then so does $\mu_{g} \circ s$ for any $g \in G$.

Given a symmetry group of an EDS $\mathcal{I}$ satisfying a number of hypothesis (see Proposition 6.1 below) the local reconstruction problem can be solved in the following way. Let $\bar{s}: V \rightarrow \bar{U}$ be an integral manifold of $\overline{\mathcal{I}}$ where $\bar{U}$ is an open subset of $\bar{M}$ on which there exists a cross-section $\sigma: \bar{U} \rightarrow M$. Now let $\gamma: V \rightarrow G$ be arbitrary and find integral manifolds $s: V \rightarrow M$ of $\mathcal{I}$ having the form

$$
s=\mu(\gamma, \sigma \circ \bar{s}) .
$$

In order that $s$ above be an integral manifold the function $\gamma$ has to satisfy a system of completely integrable differential equations. Once this system of equations for $\gamma$ are solved, the resulting $s$ is a noninvariant solution. This decomposes the problem of finding integral manifolds into two steps. First finding integral manifolds for $\overline{\mathcal{I}}$ then finding $\gamma$. Three examples are given below.

An action of $G$ on $M$ is free if $G_{x}=\{e\}$ for all $x \in M$ and we will consider the reconstruction problem for free actions only.

PROPOSITION 6.1. Let $\mathcal{I}$ be a EDS with a symmetry group $G$ which is transverse to $\mathcal{I}^{1}$ and acts freely and regularly on $M$. Let $s: N \rightarrow M$ be an integral manifold of $\mathcal{I}$. Then for every point $s\left(t_{0}\right) \in s(N)$ there exists a G-invariant open neighborhood $U$ of $s\left(t_{0}\right)$ and cross-section $\sigma: \bar{U} \rightarrow U$, such that $s(t)=\mu(\gamma(t), \sigma \circ \bar{s}(t))$ for all $t \in s^{-1}(U)$ where

1) $\bar{s}=\mathbf{q} \circ s$ is an integral manifold of $\overline{\mathcal{I}}(\bar{U})$, and

2) $\gamma: s^{-1}(U) \rightarrow G$, where the graph of $\gamma$ satisfies a completely integrable Pfaffian system on $G \times s^{-1}(U)$.

The essence of Proposition 6.1 is that every integral manifold of $\mathcal{I}$ can be locally decomposed into a solution of $\overline{\mathcal{I}}$ and a solution to a completely integral system of differential equations. We now present the examples.

Example 6.1. The EDS from Example 4.4 is

$$
\mathcal{I}=<d u-u_{x} d x, d v-v_{y} d y, d x \wedge d u_{x}, d y \wedge d v_{y}>
$$


and $G=\mathbb{R}$ acts by simultaneous translation $u \rightarrow u+a ; v \rightarrow v+a, a \in$ $G$. The quotient is

$$
\overline{\mathcal{I}}=<d w-w_{x} d x-w_{y} d y, \quad d x \wedge d w_{x}, \quad d y \wedge d w_{y}>.
$$

Let $\bar{s}:(x, y) \rightarrow \bar{M}$ be the integral manifold

$$
\bar{s}(x, y)=\left(x, y, w=f(x)+g(y), w_{x}=\partial_{x} f, w_{y}=\partial_{y} g\right)
$$

of $\overline{\mathcal{I}}$. With $\gamma(x, y)=a(x, y)$ and $\sigma$ given in (10) we have

$$
\begin{aligned}
s(x, y) & =\mu(\gamma(x, y), \sigma \circ \bar{s}(x, y)) \\
& =\left(x, y, u=f+g+a(x, y), v=a(x, y), u_{x}=\partial_{x} f, v_{x}=-\partial_{y} g\right) .
\end{aligned}
$$

This is an integral manifold if and only if

$$
\partial_{x} a=0, \quad \partial_{y} a+\partial_{y} g=0 .
$$

Solving these equations gives $a=-g(y)+c_{0}$, which makes $s$ a $G$ parameter family of (non-invariant) integral manifolds.

Example 6.2. The Pfaffian system from Example 4.3 is

$\mathcal{I}=\left\{\theta=d u-u_{x} d x-u_{y} d y, \quad \theta_{x}=d u_{x}-u_{x x} d x, \quad \theta_{y}=d u_{y}-u_{y y} d y\right\}$

on the open set $U=\subset \mathbb{R}^{7}$ given in (8). The action is $x \rightarrow x+a ; y \rightarrow$ $y+b,(a, b) \in \mathbb{R}^{2}=G$ and $\overline{\mathcal{I}}(\bar{U})$ is

$$
\overline{\mathcal{I}}(\bar{U})=<d w-w_{\alpha} d \alpha-w_{\beta} d \beta, \quad d w_{\alpha} \wedge d \alpha, \quad d w_{\beta} \wedge d \beta>.
$$

Let $\bar{s}(\alpha, \beta)=\left(\alpha, \beta, w=f(\alpha)+g(\beta), w_{\alpha}=\partial_{\alpha} f, w_{\beta}=\partial_{\beta} g\right)$ be an integral manifold of $\overline{\mathcal{I}}(\bar{U})$. Let $\gamma(\alpha, \beta)=(a(\alpha, \beta), b(\alpha, \beta))$ then $s=$ $\mu(\gamma, \sigma \circ \bar{s})$ with $\sigma$ in $(9)$ is

$$
\begin{array}{r}
s(\alpha, \beta)=(x=a(\alpha, \beta), y=b(\alpha, \beta), u=f(\alpha)+g(\beta), \\
\left.u_{x}=\alpha, u_{y}=\beta, u_{x x}=\frac{\alpha}{f^{\prime}(\alpha)}, u_{y y}=\frac{\beta}{g^{\prime}(\beta)}\right) .
\end{array}
$$

For $s(\alpha, \beta)$ to be an integral manifold of $\mathcal{I}(U)$ we get

$$
0=s^{*} \theta_{x}=d \alpha-\frac{\alpha}{f^{\prime}(\alpha)} d a \quad \text { and } \quad 0=s^{*} \theta_{y}=d \beta-\frac{\beta}{g^{\prime}(\beta)} d b .
$$

Therefore

$$
a=\int \frac{f^{\prime}(\alpha)}{\alpha} d \alpha+c_{0}, \quad b=\int \frac{b^{\prime}(\beta)}{\beta} d \beta+c_{1}
$$

This determines a $G$-parameter family of non-invariant integral manifolds. 
Example 6.3. The fourth order differential equation on pg. 156 (Bluman and Anco, 2002)

$$
u_{x x x x}=\frac{5 u_{x x} u_{x x x}}{u_{x}}+\frac{4 u_{x x}^{2}}{u}-\frac{u_{x}^{2} u_{x x}}{u^{2}}-\frac{3 u_{x} u_{x x x}}{u}-\frac{4 u_{x x}^{3}}{u_{x}^{2}}
$$

defines the completely integrable Pfaffian system

$$
\begin{aligned}
\mathcal{I}=<d u & -u_{x} d x, \quad d u_{x}-u_{x x} d x, \quad d u_{x x}-u_{x x x} d x \\
& d u_{x x x}-\left(\frac{5 u_{x x} u_{x x x}}{u_{x}}+\frac{4 u_{x x}^{2}}{u}-\frac{u_{x}^{2} u_{x x}}{u^{2}}-\frac{3 u_{x} u_{x x x}}{u}-\frac{4 u_{x x}^{3}}{u_{x}^{2}}\right) d x>.
\end{aligned}
$$

on $M=\left\{\left(x, u, u_{x}, u_{x x}, u_{x x x}\right) \in \mathbb{R}^{5} \mid u u_{x} \neq 0\right\}$. The three dimensional group $G=(a, b, c) \quad a \in \mathbb{R}, b, c \in \mathbb{R}^{*}$ with multiplication law

$$
\left(a^{\prime}, b^{\prime}, c^{\prime}\right)(a, b, c)=\left(a^{\prime}+b^{\prime} a, b^{\prime} b, c^{\prime} c\right),
$$

and action on $M$

$$
x=b x+a, u=c u, u_{x}=b^{-1} c u_{x}, u_{x x}=b^{-2} c u_{x x}, u_{x x x}=b^{-3} c u_{x x x}
$$

is a symmetry group of $\mathcal{I}$ which acts freely on $M$. The Lie algebra of infinitesimal generators on $M$ is

$$
\begin{gathered}
\Gamma=\operatorname{span}\left\{\partial_{x}, x \partial_{x}-u_{x} \partial_{u_{x}}-2 u_{x x} \partial_{u_{x x}}-3 u_{x x x} \partial_{u_{x x x}}\right. \\
\left.u \partial_{u}+u_{x} \partial_{u_{x}}+u_{x x} \partial_{u_{x x}}+u_{x x x} \partial_{u_{x x x}}\right\} .
\end{gathered}
$$

On $U=M-\left\{u u_{x} u_{x x x}+u_{x}^{2} u_{x x}-2 u u_{x x}^{2}=0\right\}$ the action is transverse to $\mathcal{I}^{1}$.

Let $\bar{U}=\left\{(t, y) \in \mathbb{R}^{2} \mid y \neq 2 t^{2}-t\right\}$ be coordinates on the quotient $U / G$ and let $\sigma(t, y)=(0,1,1, t, y)$ be a cross-section. The semi-basic one forms are

$$
\begin{aligned}
\mathcal{A}_{s b}^{1}(U)= & \operatorname{span}\left\{\left(2 u_{x x x} u u_{x}-2 u_{x x}^{2} u+u_{x x} u_{x}^{2}\right) u_{x} d u+u^{2} u_{x}^{2} d u_{x x x}\right. \\
& \left.-\left(3 u_{x x x} u u_{x}-4 u u_{x x}^{2}+2 u_{x x} u_{x}^{2}\right) u d u_{x}-\left(2 u u_{x x}-u_{x}^{2}\right) u u_{x} d u_{x x}\right\}
\end{aligned}
$$

and pulling the single generator back by $\sigma$ gives

$$
\overline{\mathcal{I}}(\bar{U})=\langle d y-(1-2 t) d t>.
$$

The most general integral manifold of $\overline{\mathcal{I}}(\bar{U})$ of the form $y(t)$ is $\bar{s}(t)=$ $\left(t, y=t-t^{2}+c_{0}\right)$, and

$$
\sigma \circ \bar{s}(t)=\left(0,1,1, t, t-t^{2}+c_{0}\right)
$$

With $\gamma: \mathbb{R} \rightarrow G$ given by $\gamma(t)=(a(t), b(t), c(t))$, we compute

$$
s(t)=\mu(\gamma(t), \sigma \circ \bar{s}(t))=\left(a(t), c(t), \frac{c(t)}{b(t)}, \frac{c(t)}{b(t)^{2}} t, \frac{c(t)}{b(t)^{3}}\left(t-t^{2}+c_{0}\right)\right) .
$$


The differential equations for $\gamma: \mathbb{R} \rightarrow G$ are obtained by writing $s^{*} \mathcal{I}=0$ and we get

$$
\dot{a}(t)=\frac{b(t)}{c_{0}-t^{2}}, \quad \dot{b}(t)=b(t) \frac{1-t}{c_{0}-t^{2}}, \quad \dot{c}(t)=\frac{c(t)}{c_{0}-t^{2}} .
$$

These differential equations are Lie equations for $\gamma: \mathbb{R} \rightarrow G$ see (Fels, 2004). The coordinates $(a, b, c)$ for $G$ have been chosen so that the reconstruction equations can be integrated by quadratures. The existence of such coordinates is guaranteed because the symmetry group is solvable.

REMARK 6.1. If $\mathcal{I}$ is a completely integrable Pfaffian system and the symmetry group $G$ acts freely and transversely to $\overline{\mathcal{I}}^{1}$ with codimension 1 orbits then then $\gamma$ in Proposition 6.1 is a solution to a Lie equation (Fels, 2004).

\section{Reduction of Exterior Differential Systems - Theoretical Aspects}

In this section we will make frequent use of the notation and results from Appendix A and B. We will assume throughout this section that $G$ is a symmetry group of the constant rank $\operatorname{EDS} \mathcal{I}$ on $M$ and that the group $G$ acts regularly on $M$.

\subsection{Transversality and Proposition 4.2}

In this subsection we look at some consequences of transversality and prove Proposition 4.2. We begin by first looking into the question of whether $\overline{\mathcal{I}}$ is a constant rank EDS. If the dimension of $I_{x}^{k} \cap \Lambda_{s b}^{k}\left(T_{x}^{*} M\right), 1 \leq$ $k \leq n$ is independent of $x$, then

$$
A_{s b}^{k}=I^{k} \cap \Lambda_{s b}^{k}\left(T^{*} M\right)
$$

is a bundle over $M$ and the set of semi-basic forms $\mathcal{A}_{s b}^{k}$ in $\mathcal{I}$ is the space of sections of $A_{s b}^{k}$.

LEMMA 7.1. If $A_{s b}^{k}$ is a bundle, then $\overline{\mathcal{I}}^{k}$ is the space of sections of the bundle $\bar{I}^{k}=\mathbf{q}^{k}\left(A_{s b}^{k}\right)$, and so $\overline{\mathcal{I}}$ is of constant rank. Conversely, if $\overline{\mathcal{I}}$ is constant rank, then $A_{s b}^{k}=\mathbf{q}^{*}\left(\bar{I}^{k}\right)$ is a bundle.

Proof. A differential form $\bar{\alpha} \in \Omega^{k}(\bar{M})$ is a section of $\bar{I}^{k}$ if and only if $\mathbf{q}^{*} \bar{\alpha} \in \mathcal{A}_{s b}^{k}$. But $\mathbf{q}^{*} \bar{\alpha} \in \Omega_{s b}^{k}(M)$ always, and so $\bar{\alpha}$ is a section of $\bar{I}^{k}=$ 
$\mathbf{q}^{k}\left(A_{s b}^{k}\right)$ if and only if $\mathbf{q}^{*} \bar{\alpha} \in \mathcal{I}^{k}$. The converse is a direct consequence of the pullback bundle construction, see Lemma A.1.

Note that the bundles $A_{s b}^{k}$ and $\bar{I}^{k}$ in Lemma 7.1 have the same rank.

In the next proposition we use Appendix B with $W=\boldsymbol{\Gamma}_{x}$ and $I=\mathcal{I}_{x}$ for $x \in M$. For example we have from equation (21) that

$p_{k}=\operatorname{dim}\left(<I_{x}^{1}>\cap \Lambda^{k}\left(T_{x}^{*} M\right)\right)=\left(\begin{array}{c}n \\ k\end{array}\right)-\left(\begin{array}{c}n-r_{1} \\ k\end{array}\right)$ and $d_{k}=r_{k}-p_{k}$,

where $d_{k}=\operatorname{rank}\left(I_{x}^{k} \bmod I_{x}^{1}\right)$.

PROPOSITION 7.1. If the symmetry group $G$ acts transversally to $\mathcal{I}^{1}$ then for $k=1, \ldots, n$ and $x \in M$

$$
\operatorname{dim} A_{s b, x}^{k}=\operatorname{dim}\left(I_{x}^{k} \cap \Lambda_{s b}^{k}\left(T_{x}^{*} M\right)\right)=\left(\begin{array}{c}
n-q \\
k
\end{array}\right)-\left(\begin{array}{c}
n-r_{1} \\
k
\end{array}\right)+d_{k},
$$

and so $A_{s b}^{k}$ is a vector bundle.

Proof. Fix $x \in M$. Using Lemma B.2 there exists a basis $\left\{\eta^{i}, \theta^{a_{1}}, \omega^{s}\right\}$, $i=1 \ldots q, a_{1}=1 \ldots r_{1}-q, s=1 \ldots n-r_{1}$ of $T_{x}^{*} M$, with $I_{x}^{1}=$ $\operatorname{span}\left\{\eta^{i}, \theta^{a_{1}}\right\}, A_{s b, x}^{1}=\operatorname{span}\left\{\theta^{a_{1}}\right\}$, and $\omega^{s} \in \Lambda_{s b}^{1}\left(T_{x}^{*} M\right)$ satisfying

$$
\eta^{i}\left(X_{j}\right)=\delta_{j}^{i}, \quad \theta^{a_{1}}\left(X_{j}\right)=0, \quad \omega^{s}\left(X_{j}\right)=0 .
$$

By Corollary B.1 we have

$$
I_{x}^{k} \cap \Lambda_{s b}^{k}\left(T_{x}^{*} M\right)=\operatorname{span}\left\{\begin{array}{l}
A_{s_{1} \ldots s_{k}}^{u_{k}} \omega^{s_{1}} \wedge \ldots \wedge \omega^{s_{k}}, \omega^{s_{1}} \wedge \ldots \wedge \omega^{s_{k-1}} \wedge \theta^{a_{1}} \\
\left., \ldots, \omega^{s_{1}} \wedge \theta^{a_{1}} \ldots \wedge \theta^{a_{k-1}}, \theta^{a_{1}} \wedge \ldots \theta^{a_{k}}\right\} .
\end{array}\right.
$$

where $A_{s_{1} \ldots s_{k}}^{u_{k}} \omega^{s_{1}} \wedge \ldots \wedge \omega^{s_{k}}$ are $d_{k}=r_{k}-p_{k}$ linearly independent $k$-forms. It is easy to compute that

$$
\operatorname{dim} A_{s b, x}^{k}=\operatorname{dim} I_{x}^{k} \cap \Lambda_{s b}^{k}\left(T_{x}^{*} M\right)=\left(\begin{array}{c}
n-q \\
k
\end{array}\right)-\left(\begin{array}{c}
n-r_{1} \\
k
\end{array}\right)+d_{k}
$$

which is independent of $x$.

COROLLARY 7.1. Let $G$ be a symmetry group of the constant rank $E D S \mathcal{I}$. If $G$ acts transversally to $\mathcal{I}^{1}$ then $\overline{\mathcal{I}}$ is a constant rank EDS.

If we introduce the ideal

$$
\mathcal{I}_{\mathcal{A}}=<\mathcal{A}_{s b}>=<\mathcal{A}_{s b}^{1}, \mathcal{A}_{s b}^{2}, \ldots, \mathcal{A}_{s b}^{n}>
$$

we have the following corollaries (See Corollary A.1 and A.2). 
COROLLARY 7.2. Suppose $U$ is an open set in $M$ such that $A_{s b}^{k}(U), k=$ $1, \ldots, n$ are bundles, and let $\bar{U}=\mathbf{q}(U)$. If

$\left\{\bar{\theta}^{a_{1}}\right\}$ is a basis for $\overline{\mathcal{I}}^{1}(\bar{U})$,

$\left\{\bar{\alpha}^{a_{2}}\right\}$ is a basis for $\overline{\mathcal{I}}^{2}(\bar{U})$,

$\vdots$

$\{\bar{\nu}\}^{a_{n}}$ is a basis for $\overline{\mathcal{I}}^{n}(\bar{U})$

then $\mathcal{I}_{\mathcal{A}}(U)=\left\langle\mathbf{q}^{*} \bar{\theta}^{a_{1}}, \mathbf{q}^{*} \bar{\alpha}^{a_{2}}, \ldots, \mathbf{q}^{*} \bar{\nu}^{a_{n}}\right\rangle=\left\langle\mathbf{q}^{*} \overline{\mathcal{I}}(\bar{U})\right\rangle$.

We now proceed to the proof of Proposition 4.2.

Proof. By Proposition 7.1 the bundles $A_{s b}^{k}$ and $\bar{I}^{k}$ are well defined $\overline{\mathcal{I}}$ is a constant rank EDS. Now let $x \in M$ and $U$ be an open set about $x$ where $\left\{X_{i}\right\}, i=1 \ldots q$ are a basis for $\boldsymbol{\Gamma}(U)$ and $\left\{\eta^{i}, \theta^{w_{1}}, \omega^{s}\right\}, i=$ $1 \ldots q, w_{1}=1 \ldots r_{1}-q, s=1 \ldots n-r_{1}$ are a basis for $T^{*} U$ and where the forms $\left\{\eta^{i}, \theta^{w_{1}}\right\}$ form a basis for $\mathcal{I}^{1}(U)$ and satisfy the conditions from Lemma B.2

$$
\eta^{i}\left(X_{j}\right)=\delta_{j}^{i}, \quad \theta^{w_{1}}\left(X_{j}\right)=0, \quad \omega^{s}\left(X_{j}\right)=0,
$$

on $U$. Construct the generators for $\mathcal{I}(U)$ and $\mathcal{I}_{\mathcal{A}}(U)$ exactly as in Lemma B.3 and Corollary B.1 (a refinement of $U$ might be necessary). That is

$$
\mathcal{I}(U)=<\eta^{i}, \theta^{w_{1}}, A_{s_{1} s_{2}}^{u_{2}} \omega^{s_{1}} \wedge \omega^{s_{2}}, \ldots, A_{s_{1} \ldots s_{n-r_{1}}}^{u_{n-r_{1}}} \omega^{s_{1}} \wedge \ldots \wedge \omega^{s_{n-r_{1}}}>
$$

and

$$
\mathcal{I}_{A}(U)=<\theta^{w_{1}}, A_{s_{1} s_{2}}^{u_{2}} \omega^{s_{1}} \wedge \omega^{s_{2}}, \ldots, A_{s_{1} \ldots s_{n-r_{1}}}^{u_{n-r_{1}}} \omega^{s_{1}} \wedge \ldots \wedge \omega^{s_{n-r_{1}}}>.
$$

where $A_{r_{1} \ldots r_{l}}^{u_{l}} \omega^{r_{1}} \wedge \ldots \wedge \omega^{r_{l}}, u_{l}=1 \ldots d_{l}$ are point-wise linearly independent.

We now use the same argument as in Lemma B.5. The condition that $\mathcal{I}^{1}(U) \ldots \mathcal{I}^{k}(U)$ generate $\mathcal{I}(U)$ (after perhaps refining $U$ ) imply that for all $l>k$ there exists $\kappa_{u_{k}}^{u_{l}} \in \Omega^{l-k}(U)$ such that

$A_{r_{1} \ldots r_{l}}^{u_{l}} \wedge \omega^{r_{1}} \wedge \ldots \wedge \omega^{r_{l}}=\kappa_{u_{k}}^{u_{l}} \wedge\left(A_{r_{1} \ldots r_{k}}^{u_{k}} \omega^{r_{1}} \wedge \ldots \wedge \omega^{r_{k}}\right)+\ldots+\kappa_{u_{1}}^{u_{l}} \wedge\left(A_{r_{2}}^{u_{l}} \omega^{r_{2}}\right)$.

Therefore

$$
\mathcal{I}_{\mathcal{A}}(U)=<\theta^{w_{1}}, A_{s_{1} s_{2}}^{u_{2}} \omega^{s_{1}} \wedge \omega^{s_{2}}, \ldots, A_{s_{1} \ldots s_{k}}^{k} \omega^{s_{1}} \wedge \ldots \wedge \omega^{s_{k}}>,
$$

with $\mathcal{I}(U)$ behaving similarly.

Now choose forms $\alpha^{w_{2}} \in \mathcal{A}_{s b}^{2}(U), \ldots, \kappa^{w_{k}} \in \mathcal{A}_{s b}^{k}(U)$ which provide a basis for $\mathcal{A}_{s b}^{2}(U) \bmod \mathcal{A}_{s b}^{1}(U), \ldots, \mathcal{A}_{s b}^{k}(U) \bmod \mathcal{A}_{s b}^{k-1}(U)$. These forms along with $\eta^{i}$ and $\theta^{w_{1}}$ satisfy the conditions of the proposition.

One consequence of Proposition 4.2 and Corollary 4.1 is the next corollary. 
COROLLARY 7.3. Let $\mathcal{I}$ be an EDS algebraically generated by it first $k$-homogeneous components and let $G$ a Lie symmetry group which acts transversally to $\mathcal{I}$. Then $\overline{\mathcal{I}}$ and $\mathcal{I}_{A}$ are generated their first $k$ homogeneous components, and for $k \geq 2$

$$
\operatorname{rank}\left(\overline{\mathcal{I}}^{k} \bmod \overline{\mathcal{I}}^{k-1}\right)=\operatorname{rank}\left(\mathcal{I}^{k} \bmod \mathcal{I}^{k-1}\right)=\operatorname{rank}\left(\mathcal{I}_{\mathcal{A}}^{k} \bmod \mathcal{I}_{\mathcal{A}}^{k-1}\right) .
$$

\subsection{Pfaffian Systems And TheOrem 5.1}

We now turn towards proving Theorem 5.1 on the reduction of Pfaffian systems. We begin by rewriting Corollary 7.4 when $\mathcal{I}$ is a Pfaffian.

COROLLARY 7.4. Let $\mathcal{I}$ be a Pfaffian system and $G$ a Lie symmetry group which acts transversally to $\mathcal{I}^{1}$. Then $\mathcal{I}_{\mathcal{A}}$ and $\overline{\mathcal{I}}$ are algebraically generated by their first two homogeneous components.

This corollary does not say that the reduced system is a Pfaffian system. The next lemma allows us to decide when a reduced Pfaffian system $\overline{\mathcal{I}}$ is a Pfaffian system in terms of $\mathcal{I}_{\mathcal{A}}$.

LEMMA 7.2. Let $\mathcal{I}$ be a Pfaffian system and $G$ a Lie symmetry group. The reduced EDS $\overline{\mathcal{I}}$ is a constant rank Pfaffian system if and only if $\mathcal{I}_{\mathcal{A}}$ is a constant rank Pfaffian system.

Proof. Let $U \subset M, \bar{U} \subset \bar{M}$, and $\sigma: \bar{U} \rightarrow U$ satisfy the conditions of Corollary 4.1. By Corollary 7.2 $\mathcal{I}_{\mathcal{A}}(U)=\left\langle\mathbf{q}^{*} \overline{\mathcal{I}}(\bar{U})\right\rangle$, so if $\overline{\mathcal{I}}$ is a Pfaffian system then $\mathcal{I}_{\mathcal{A}}$ is a Pfaffian system. By Corollary $4.1 \overline{\mathcal{I}}(U)=$ $\sigma^{*} \mathcal{I}_{\mathcal{A}}(U)$, therefore if $\mathcal{I}_{\mathcal{A}}$ is a Pfaffian system, then $\overline{\mathcal{I}}$ is a Pfaffian system.

The main result on Pfaffian systems from section 5 can now be proved.

Proof. (Theorem 5.1) We begin by proving the only if condtion. The initial conditions are $I$ is of rank $r, I^{\prime}$ is of rank $r^{\prime}$, and the orbits of $G$ are of dimension $q$. Suppose that $I^{\perp} \cap \boldsymbol{\Gamma}=0$, but $\operatorname{dim}\left(\left(I^{\prime}\right)^{\perp} \cap \boldsymbol{\Gamma}\right)=t \geq 1$. We will show that $\mathcal{I}_{\mathcal{A}}$ is not a Pfaffian system, and so by Lemma 7.3 $\overline{\mathcal{I}}$ is not. Let $U$ be an open set with $\left\{X_{u}\right\}, u=1 \ldots t$ be a basis for $\left(I^{\prime}\right)^{\perp} \cap \boldsymbol{\Gamma}$ and complete this to a basis $\left\{X_{u}, X_{\mu}\right\}, \mu=1 \ldots q-t$ for $\boldsymbol{\Gamma}(U)$. Choose a local basis $\left\{\eta^{\nu}, \theta^{z}\right\}, \nu=1 \ldots q-t, z=1 \ldots r^{\prime}-t$ for $\left(\mathcal{I}^{\prime}\right)^{1}(U)$ such that (see Lemma B.2)

$$
\eta^{\nu}\left(X_{u}\right)=0, \eta^{\nu}\left(X_{\mu}\right)=\delta_{\mu}^{\nu}, \theta^{z}\left(X_{u}\right)=0, \theta^{z}\left(X_{\mu}\right)=0 .
$$

Complete this to a local basis $\left\{\eta^{v}, \theta^{a}, \eta^{\nu}, \theta^{z}\right\}, v=1 \ldots t, a=1 \ldots r-$ $r^{\prime}-t$ for $\mathcal{I}^{1}(U)$ such that

$$
\eta^{v}\left(X_{u}\right)=\delta_{u}^{v}, \eta^{v}\left(X_{\mu}\right)=0, \theta^{a}\left(X_{u}\right)=0, \theta^{a}\left(X_{\mu}\right)=0,
$$


and finally complete this to a coframe on $U$ with $\omega^{s}, s=1 \ldots n-r$ where $\omega^{s}(X)=0$ for all $X \in \boldsymbol{\Gamma}(U)$. Now

$$
\mathcal{A}_{s b}^{1}(U)=\operatorname{span}\left\{\theta^{a}, \theta^{z}\right\}
$$

and the structure equations in our coframe are

$$
\begin{array}{lll}
d \eta^{\nu} \equiv d \theta^{z} \equiv 0 & \bmod \mathcal{I}^{1}(U) \\
d \eta^{v} \equiv A_{s t}^{v} \omega^{s} \wedge \omega^{t} & \bmod \mathcal{I}^{1}(U) \\
d \theta^{a} \equiv A_{s t}^{a} \omega^{s} \wedge \omega^{t} & \bmod \mathcal{I}^{1}(U)
\end{array}
$$

where $A_{s t}^{v} \omega^{s} \wedge \omega^{t}$ and $A_{s t}^{a} \omega^{s} \wedge \omega^{t}$ are point-wise linearly independent, otherwise some combination of $\eta^{v}$ and $\theta^{a}$ would be in $\mathcal{I}^{\prime}(U)$, which is a contradiction. Therefore

$$
\mathcal{A}_{s b}^{2}(U)=\operatorname{span}\left\{A_{s t}^{v} \omega^{s} \wedge \omega^{t}, A_{s t}^{a} \omega^{t} \wedge \omega^{t}, \rho_{a} \wedge \theta^{a}, \rho_{z} \wedge \theta^{z}\right\},
$$

where $\rho_{a}, \rho_{z} \in \Omega_{s b}^{1}(U)$. By equations (11) and (12) the terms $A_{s t}^{v} \omega^{s} \wedge$ $\omega^{t} \in \mathcal{A}_{s b}^{2}(U)$ are not in the ideal $\left\langle\mathcal{I}_{\mathcal{A}}^{1}(U), d \mathcal{I}_{\mathcal{A}}^{1}(U)>\right.$ and so $\mathcal{I}_{\mathcal{A}}$ is not a Pfaffian system. Therefore by Lemma $7.3 \overline{\mathcal{I}}$ is not a Pfaffian system which proves the only if condition.

We now prove that if $I^{\perp} \cap \boldsymbol{\Gamma}=0$ and $\operatorname{dim}\left(\left(I^{\prime}\right)^{\perp} \cap \boldsymbol{\Gamma}\right)=0$ then $\mathcal{I}_{\mathcal{A}}$ is a Pfaffian system and by Lemma 7.3 , so is $\overline{\mathcal{I}}$. We construct the coframe exactly as in the case above, where $t$ would be zero and so there no $X_{u}$ vector-fields, or $\eta^{v}$ forms.

The structure equations in (12) are the same except that the second equation in (12) is not there. Therefore the generating forms for $\mathcal{I}(U)$ are given by

$$
\begin{aligned}
& \mathcal{I}^{1}(U)=\operatorname{span}\left\{\theta^{z}, \eta^{\nu}, \theta^{a}\right\}, \\
& \mathcal{I}^{2}(U)=\operatorname{span}\left\{A_{r s}^{a} \omega^{r} \wedge \omega^{s}, \rho_{a} \wedge \theta^{a}, \rho_{\nu} \wedge \eta^{\nu}, \rho_{z} \wedge \theta^{z}\right\} .
\end{aligned}
$$

The semi-basic forms are

$$
\mathcal{A}_{s b}^{1}(U)=\operatorname{span}\left\{\theta^{z}, \theta^{a}\right\}, \mathcal{A}_{s b}^{2}(U)=\operatorname{span}\left\{A_{r s}^{a} \omega^{r} \wedge \omega^{s}, \rho_{a} \wedge \theta^{a}, \rho_{z} \wedge \theta^{z}\right\} .
$$

We now show that the last equation in (12) can be refined to

$$
d \theta^{a}=A_{s t}^{a} \omega^{s} \wedge \omega^{t} \quad \bmod \mathcal{A}_{s b}^{1}(U) .
$$

This will prove that $\mathcal{I}_{\mathcal{A}}$ is a Pfaffian system. Expanding out the last equation in (12) we have

$$
d \theta^{a}=A_{s t}^{a} \omega^{s} \wedge \omega^{t}+P_{s \nu}^{a} \omega^{s} \wedge \eta^{\nu}+Q_{\mu \nu}^{a} \eta^{\mu} \wedge \eta^{\nu} \quad \bmod \mathcal{A}_{s b}^{1}(U) .
$$

Now using the invariance property $\mathcal{L}_{X_{\mu}} \theta^{a}=\iota_{X_{\mu}} d \theta^{a}=0 \bmod \mathcal{I}^{1}$ we have $P_{s \mu}^{\alpha}=0$. Then using $d \theta^{a}\left(X_{\mu}, X_{\nu}\right)=-\theta^{a}\left(\left[X_{\mu}, X_{\nu}\right]\right)=0$ we have 
$Q_{\mu \nu}^{a}=0$, and so (13) holds. This proves that $\mathcal{I}_{\mathcal{A}}$ is a Pfaffian system and hence by Lemma 7.2 so is $\overline{\mathcal{I}}$. The rank of $\overline{\mathcal{I}}$ is clearly $r-q$, while the fact that $\overline{\mathcal{I}^{\prime}}=(\overline{\mathcal{I}})^{\prime}$ is left as an exercise.

\subsection{Reconstruction and Proposition 6.1}

In this subsection we give the proof of Proposition 6.1.

We start with a useful construction. Suppose that $G$ is a Lie group which acts regularly and freely on $M$. Let $\bar{U} \subset \bar{M}$ be an open set on which there exists a cross section $\sigma: \bar{U} \rightarrow U$ where $U=\mathbf{q}^{-1}(\bar{U})$. Define the function $\Phi: G \times \bar{U} \rightarrow U$ by

$$
\Phi(g, \bar{x})=\mu(g, \sigma(\bar{x})) .
$$

The function $\Phi$ is clearly one-to-one and onto because $G$ acts freely, but $\Phi$ is also a diffeomorphism. This can be checked by a computation similar to the one in (16). The inverse $\Phi^{-1}: U \rightarrow G \times \bar{U}$ may be written

$$
\Phi^{-1}(x)=(\lambda(x), \mathbf{q}(x)),
$$

where the function $\lambda$ is $G$ equivariant. By applying $\Phi$ to (14) we then have the following identity on the open set $U \subset M$,

$$
x=\mu(\lambda(x), \sigma \circ \mathbf{q}(x)) \text { for all } x \in U .
$$

REMARK 7.1. $\left(U, \Phi^{-1}\right)$ is a local trivialization of the principle bundle $M \rightarrow \bar{M}$.

We can use the construction above to give a local factor theorem for immersions into $M$.

LEMMA 7.3. Let $G$ act regularly and freely on $M$ and let $s: N \rightarrow M$ be an immersion. Then about each point $s\left(t_{0}\right) \in s(N)$ there exists a $G$-invariant neighborhood $U$ of $s\left(t_{0}\right)$ with cross-section $\sigma: \bar{U} \rightarrow U$, and a smooth function $\gamma: s^{-1}(U) \rightarrow G$ such that for $t \in s^{-1}(U)$ we have

$$
s(t)=\mu(\gamma(t), \sigma \circ \mathbf{q} \circ s(t)) .
$$

Proof. Let $U$ be a $G$-invariant neighborhood of $s\left(t_{0}\right)$ with cross-section $\sigma: \bar{U} \rightarrow U$ where $\bar{U}=\mathbf{q}(U)$ and let $\lambda: U \rightarrow G$ come from the local trivialization $\Phi^{-1}: U \rightarrow G \times \bar{U}$ as in equation (14). Then by equation (15)

$$
s(t)=\mu(\lambda \circ s(t), \sigma \circ \mathbf{q} \circ s(t)) \quad \text { for all } t \in s^{-1}(U),
$$

so with $\gamma=\lambda \circ s(t)$ the lemma is proved. 
We are now in a position to prove Proposition 6.1.

Proof. (Proposition 6.1). By applying Lemma 7.3 in the case of an integral manifold of an EDS we get a factor theorem for integral manifolds which proves everything in Proposition 6.1 except the fact that the graph of $\gamma$ is an integral manifold of a completely integral Pfaffian system on $G \times V$. Starting with the integral manifold $s, U, \bar{U}$ and $\sigma$ as in Lemma 7.3 let $\bar{s}=\mathbf{q} \circ s$ and $\hat{s}=\sigma \circ \bar{s}$ and define the function $\Psi: G \times V \rightarrow U$ by

$$
\Psi(g, t)=\mu(g, \hat{s}(t)) .
$$

It is easy to see that if $s$ is an integral manifold that the graph of $\gamma$ is an integral manifold to $\Psi^{*}(\mathcal{I}(U))$. Therefore to prove the last part of the proposition we need to show that $\Psi^{*}(\mathcal{I}(U))$ is a completely integrable Pfaffian system on $G \times V$. This is a local statement so we can assume by Proposition 4.2 that

$$
\mathcal{I}(U)=<\eta^{i}, \theta^{w_{1}}, \alpha^{w_{2}}, \ldots, \kappa^{w_{k}}>
$$

with the conditions on the forms as stated in the theorem. We have assumed the action is free so these are easily shown to exist on a $G$ invariant open set which we assume to be $U$.

We now pull back the generators for $\mathcal{I}(U)$ by $\Psi^{*}$. Let $T \in T_{t} V$ and $R_{g} \in T_{g} G$, where $R_{g}$ is a right invariant vector field at $g$. We find

$$
\begin{aligned}
\Psi^{*} \theta^{w_{1}}\left(R_{g}, T\right) & =\left(\mu_{\hat{s}}^{*} \theta^{w_{1}}\right)\left(R_{g}\right)+\left(\hat{s}^{*} \mu_{g}^{*} \theta^{w_{1}}\right)(T) \\
& =\theta^{w_{1}}\left(X_{\mu(g, \hat{s})}\right)+\mu_{g}^{*} \theta^{w_{1}}\left(\hat{s}_{*} T\right),
\end{aligned}
$$

where $X_{\mu(g, \hat{s})}$ is the infinitesimal generator corresponding to $R_{e}$. The first term is clearly 0 because $\theta^{w_{1}}$ is semi-basic. The second term can be written as

$$
\left(\mu_{g}^{*} \theta^{w_{1}}\right)\left(\hat{s}_{*} T\right)=\left(\sigma^{*} \mu_{g}^{*} \theta^{w_{1}}\right)\left(\bar{s}_{*} T\right) .
$$

Now $\mu_{g}^{*} \theta^{w_{1}}$ is semi-basic, so, by Lemma 4.1 and the fact that $\bar{s}$ is an integral manifold of $\overline{\mathcal{I}}(\bar{U})$, this term is 0 . A similar argument shows $\Psi^{*} \alpha=\mu_{\hat{s}}^{*} \alpha+\hat{s}^{*} \mu_{g}^{*} \alpha=0$ for all $\alpha \in \mathcal{A}_{s b}(U)$. Therefore

$$
<\Psi^{*}(\mathcal{I}(U))>=<\Psi^{*} \eta^{i}>.
$$

Now $\left\langle\Psi^{*}(\mathcal{I}(U))>\right.$ is easily checked to be a differential ideal, and so $\left.d \Psi^{*} \eta^{i} \in<\Psi^{*} \eta^{i}\right\rangle$ which proves that $\left\langle\Psi^{*} \eta^{i}\right\rangle$ is completely integrable. 


\section{Appendix}

\section{A. G basic differential forms}

Let $G$ be a Lie group acting regularly on $M$ with $q$-dimensional orbits and projection map q : $M \rightarrow \bar{M}$. In this appendix we characterize the pullback $\mathbf{q}^{*} \Omega^{*}(\bar{M}) \subset \Omega^{*}(M)$ which is a subalgebra of $\Omega^{*}(M)$ over $\mathbb{R}$.

Recall the Lie algebra of infinitesimal generators is denoted by $\Gamma$ and the corresponding integrable distribution is $\Gamma \subset T M$. Any $\bar{\alpha} \in \Omega^{k}(\bar{M})$ satisfies

$$
\iota_{X}\left(\mathbf{q}^{*} \bar{\alpha}\right)=0 \quad \text { for all } \quad X \in \boldsymbol{\Gamma} .
$$

This observation motivates the definition of the semi-basic $k$-forms $\Lambda_{s b}^{k}\left(T^{*} M\right) \subset \Lambda^{k}\left(T^{*} M\right)$ given point-wise by

$$
\Lambda_{s b}^{k}\left(T_{x}^{*} M\right)=\left\{\alpha \in \Lambda^{k}\left(T_{x}^{*} M\right) \mid \iota_{X} \alpha=0, \quad \text { for all } X \in \boldsymbol{\Gamma}_{x}\right\} .
$$

We then have the following important lemma.

LEMMA A.1. The subset $\Lambda_{s b}^{k}\left(T^{*} M\right) \subset \Lambda^{k}\left(T^{*} M\right)$ defined by

$$
\Lambda_{s b}^{k}\left(T^{*} M\right)=\bigcup_{x \in M} \Lambda_{s b}^{k}\left(T_{x}^{*} M\right)
$$

is a $G$ invariant vector sub-bundle that is isomorphic to the pullback bundle $\mathbf{q}^{*}\left(\Lambda^{k}\left(T^{*} \bar{M}\right)\right)$. The rank of $\Lambda_{s b}^{k}\left(T^{*} M\right)$ is $\left(\begin{array}{c}n-q \\ k\end{array}\right)$. This gives rise to the commutative diagram

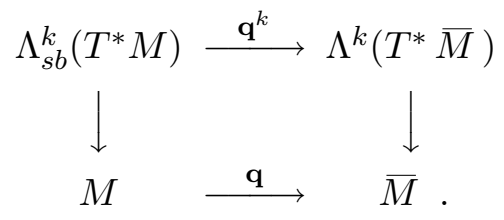

of vector bundles and $\mathbf{q}^{k}$ is a bundle homomorphism which is an isomorphism on the fibres.

Let $\Omega_{s b}^{k}(M)$ be the sections of $\Lambda_{s b}^{k}\left(T^{*} M\right)$ which are called the semibasic differential $k$-forms. We give a local coordinate form for $\alpha \in$ $\Omega_{s b}^{k}(M)$. First for any point $x \in M$ there exists a neighborhood $U \subset M$ with $\bar{U}=\mathbf{q}(U)$ and local coordinates $\left(x^{i}, y^{a}\right), i=1 \ldots q, a=1 \ldots n-q$ on $U$ and local coordinates $\bar{y}^{a}$ on $\bar{U}$ such that $\mathbf{q}: U \rightarrow \bar{U}$ has the coordinate form $\bar{y}^{a}=\mathbf{q}^{a}\left(x^{i}, y^{a}\right)=y^{a}$. The set $U$ with coordinates $\left(x^{i}, y^{a}\right)$ is called a $G$-adapted coordinate neighborhood. In a $G$-adapted coordinate neighbourhood $U, \alpha \in \Omega_{s b}^{k}(U)$ has the form

$$
\alpha=\rho_{a_{1} a_{2} \ldots a_{k}}\left(x^{i}, y^{a}\right) d y^{a_{1}} \wedge d y^{a_{2}} \wedge \ldots \wedge d y^{a_{k}} .
$$

where $\rho_{a_{1} a_{2} \ldots a_{k}}\left(x^{i}, y^{a}\right) \in C^{\infty}(U)$, and $1 \leq a_{1}, \ldots a_{k} \leq n-q$. 
LEMMA A.2. Let $U$ be an open set such that $\mathbf{q}: U \rightarrow \bar{U}$, with $\bar{U}=$ $\mathbf{q}(U)$ and cross-section $\sigma: \bar{U} \rightarrow U$. If $\alpha \in \Omega_{s b}^{k}(M)$ then

$$
\left(\mathbf{q}^{*} \sigma^{*} \alpha\right)_{\sigma(\bar{x})}=\alpha_{\sigma(\bar{x})} \text { for all } \bar{x} \in \bar{U} .
$$

Proof. Both sides of the equation are semi-basic forms and so they are completely determined by their values on an $n-q$ dimensional subspace of $T_{\sigma(\bar{x})}^{*} U$ which is complementary to $\boldsymbol{\Gamma}_{x}=\operatorname{Vert}_{x} M$. The subspace $\sigma_{*} T_{\bar{x}} \bar{U}$ is complementary and we find for $\bar{v} \in T_{\bar{x}} \bar{U}$,

$$
\left(\mathbf{q}^{*} \sigma^{*} \alpha\right)_{\sigma(\bar{x})}\left(\sigma_{*}(\bar{v})\right)=\sigma^{*} \alpha_{\sigma(\bar{x})}(\bar{v})=\alpha_{\sigma(\bar{x})}\left(\sigma_{*} \bar{v}\right),
$$

which proves the lemma.

The following two corollaries of Lemma A.1 are direct consequences of the pullback bundle construction.

COROLLARY A.1. If $\bar{U} \subset \bar{M}$ is an open set and $\left\{\bar{\kappa}^{a_{k}}\right\}$ a basis of sections for $\left.\Omega^{k}(\bar{U})\right\}$, then $\left\{\mathbf{q}^{*} \bar{\kappa}^{a_{k}}\right\}$ is a basis of sections for $\Lambda_{s b}^{k}\left(T^{*} U\right)$ where $U$ is any open set in $M$ such that $\mathbf{q}(U)=\bar{U}$.

COROLLARY A.2. Let $U \subset M$ be an open set which admits a crosssection $\sigma: \bar{U} \rightarrow U$ where $\bar{U}=\mathbf{q}(U)$. If $\left\{\kappa^{a_{k}}\right\}$ is a basis of sections for $\Lambda_{s b}^{k}\left(T^{*} U\right)$, then $\left\{\sigma^{*} \kappa^{a_{k}}\right\}$ is a basis of sections of $\Lambda^{k}\left(T^{*} \bar{U}\right)$ and $\sigma^{*} \kappa^{a_{k}}=\mathbf{q}^{k} \circ \kappa^{a_{k}} \circ \sigma$.

Note that every $G$-adapted coordinate neighborhood $U$ satisfies the conditions of Corollaries A.1 and A.2 with $\bar{U}=\mathbf{q}(U)$.

Finally we can characterize the subset $\mathbf{q}^{*}\left(\Omega^{*}(\bar{M})\right) \subset \Omega^{*}(M)$ by the following lemma.

LEMMA A.3. Let $\alpha \in \Omega^{k}(M)$, then $\alpha \in \mathbf{q}^{*}\left(\Omega^{k}(\bar{M})\right)$ if and only if

$\alpha \in \Omega_{s b}^{k}(M)^{G}$. In this case there exists a unique $\bar{\alpha} \in \Omega^{k}(\bar{M})$ such that $\alpha=\mathbf{q}^{*} \bar{\alpha}$.

The lemma shows that

$$
\mathbf{q}^{*}\left(\Omega^{*}(\bar{M})\right)=\Omega_{s b}^{*}(M)^{G} .
$$

The differential forms $\Omega_{s b}^{*}(M)^{G} \subset \Omega_{s b}^{*}(M)$ are the $G$ basic differential forms on $M$.

\section{B. Algebraic Results}

Let $V$ be an $n$-dimensional real vector space and let $\Lambda^{*}\left(V^{*}\right)$ the exterior algebra of forms. Let $I \subset \Lambda^{*}\left(V^{*}\right)$ be an ideal, $I^{k}=I \cap \Lambda^{k}\left(V^{*}\right)$ be the elements of degree $k$ in $I$, and $r_{k}=\operatorname{dim} I^{k}$. If $A \subset \Lambda^{*}\left(V^{*}\right)$ then $\langle A>$ denotes the ideal generated by $A$, and $\bmod A$ denotes modulo the ideal $<A>$. 
LEMMA B.1. If $k>n-r^{1}$ then $I^{k} \equiv 0 \bmod I^{1}$.

Proof. Let $\left\{\theta^{a}\right\}, a=1 \ldots r^{1}$ be a basis for $I^{1}$, and complete this to a basis $\left\{\theta^{a}, \omega^{s}\right\}, s=1 \ldots n-r_{1}$ for $V^{*}$. If $\alpha \in \Lambda^{k}\left(V^{*}\right)$ then in this basis

$$
\begin{aligned}
\alpha= & A_{a_{1} \ldots a_{k}} \theta^{a_{1}} \wedge \ldots \wedge \theta^{a_{k}}+A_{s_{1} a_{1} \ldots a_{k-1}} \omega^{s_{1}} \wedge \theta^{a_{1}} \wedge \ldots \wedge \theta^{a_{k-1}}+\ldots+ \\
& A_{s_{1} \ldots s_{l} a_{1} \ldots a_{k-l}} \omega^{s_{1}} \wedge \ldots \wedge \omega^{s_{l}} \wedge \theta^{a_{1}} \wedge \ldots \wedge \theta^{a_{k-l}}+\ldots+ \\
& A_{s_{1} \ldots s_{k}} \omega^{s_{1}} \wedge \ldots \wedge \omega^{s_{k}} .
\end{aligned}
$$

If $k>n-r_{1}$, there are no terms involving just wedge products of only $\omega^{s}$.

For each $k=2, \ldots, n-r_{1}$, define the integers $p_{k}=\operatorname{dim}\left(<I^{1}>^{k}\right)$ and $d_{k}=\operatorname{dim}\left(I^{k} \bmod I^{1}\right)$ then

$$
p_{k}=\left(\begin{array}{c}
n \\
k
\end{array}\right)-\left(\begin{array}{c}
n-r_{1} \\
k
\end{array}\right), \text { and } \quad d_{k}=r_{k}-p_{k}
$$

DEFINITION B.1. A subspace $W \subset V$ is transverse to $I^{1}$ if $\left(I^{1}\right)^{\perp} \cap$ $W=0$.

LEMMA B.2. Let $W \subset V$ be a q-dimensional subspace which is transverse to $I^{1}$. Let $\left\{X_{j}\right\}, j=1 \ldots q$ be a basis for $W$, then there exists a basis $\left\{\eta^{i}, \theta^{a_{1}}, \omega^{s}\right\}, i=1 \ldots q, a_{1}=1 \ldots r_{1}-q, s=1 \ldots n-r_{1}$ for $V^{*}$ with $\left\{\eta^{i}, \theta^{a_{1}}\right\}$ a basis for $I^{1}$ such that

$$
\eta^{i}\left(X_{j}\right)=\delta_{j}^{i}, \quad \theta^{a_{1}}\left(X_{j}\right)=0, \quad \omega^{s}\left(X_{j}\right)=0 .
$$

Proof. Exercise.

LEMMA B.3. Let $W \subset V$ be a q-dimensional subspace which is transverse to $I^{1}$ and let $\eta^{i}, \theta^{a_{1}}, \omega^{r}$ be the basis for $V^{*}$ from Lemma B.2. For all $k \in \mathbb{Z} 2 \leq k \leq n-r_{1}$ there exists linearly independent $k$-forms $A_{s_{1} \ldots s_{k}}^{u_{k}} \omega^{s_{1}} \wedge \ldots \wedge \omega^{s_{k}}, 1 \leq u_{k} \leq d_{k}$ such that

$$
I^{k}=\operatorname{span}\left\{A_{s_{1} \ldots s_{k}}^{u_{k}} \omega^{s_{1}} \wedge \ldots \wedge \omega^{s_{k}}, \rho_{i} \wedge \eta^{i}, \tau_{a_{1}} \wedge \theta^{a_{1}}\right\}
$$

where $\rho_{i}, \tau_{a_{1}} \in \Lambda^{k-1}\left(V^{*}\right)$ are arbitrary.

Proof. The dimension of $I^{k} \bmod I^{1}$ is $d_{k}$ and since $\left\{\eta^{i}, \theta^{a_{1}}\right\}$ is a basis for $I^{1}$ there exists representatives for a basis of $I^{k} \bmod I^{1}$ of the form $A_{s_{1} \ldots s_{k}}^{u_{k}} \omega^{s_{1}} \wedge \ldots \wedge \omega^{s_{k}}, u_{k}=1 \ldots d_{k}$. Therefore if $\beta \in I^{k}$ there exists $B_{u_{k}} \in \mathbb{R}$ and $\mu_{i}, \nu_{a_{1}} \in \Lambda^{k-1}\left(V^{*}\right)$ such that

$$
\beta=B_{u_{k}} A_{s_{1} \ldots s_{k}}^{u_{k}} \omega^{s_{1}} \wedge \ldots \wedge \omega^{s_{k}}+\mu_{i} \wedge \eta^{i}+\nu_{a_{1}} \wedge \theta^{a_{1}},
$$

which proves the theorem.

Given a subspace $W \subset V$ define the subalgebra $\Lambda_{W}^{*}\left(V^{*}\right)=\{\alpha \subset$ $\Lambda^{*}\left(V^{*}\right) \mid \iota_{X} \alpha=0$, for all $\left.X \in W\right\}$. 
LEMMA B.4. $\Lambda_{W}^{k}\left(V^{*}\right) \cong \Lambda^{k}\left(\Lambda_{W}^{1}\left(V^{*}\right)\right)$.

Let $A=I \cap \Lambda_{W}^{*}\left(V^{*}\right)$ which is a subalgebra of $\Lambda^{*}(V)$, whose degree $k$ elements are

$$
A^{k}=I^{k} \cap \Lambda_{W}^{k}\left(V^{*}\right) .
$$

The subalgebra $A$ generates the ideal $I_{A}=\langle A\rangle$. Now notice that the forms in Lemma B.3 satisfy $A_{s_{1} \ldots s_{k}}^{u_{k}} \omega^{s_{1}} \wedge \ldots \wedge \omega^{s_{k}} \in I^{k} \cap \Lambda_{W}^{k}\left(V^{*}\right)=A^{k}$ which leads to the second part of the next Corollary.

COROLLARY B.1. Using the basis for $V^{*}$ from Lemma B. 2 and the forms from Lemma B.3 we have

$$
I=<\eta^{i}, \theta^{a_{1}}, A_{s_{1} s_{2}}^{u_{2}} \omega^{s_{1}} \wedge \omega^{s_{2}}, \ldots, A_{s_{1} \ldots s_{n-r_{1}}}^{u_{n-r_{1}}} \omega^{s_{1}} \wedge \ldots \wedge \omega^{s_{n-r_{1}}}>
$$

and

$$
I_{A}=<\theta^{a_{1}}, A_{s_{1} s_{2}}^{u_{2}} \omega^{s_{1}} \wedge \omega^{s_{2}}, \ldots, A_{s_{1} \ldots s_{n-r_{1}}}^{u_{n-r_{1}}} \omega^{s_{1}} \wedge \ldots \wedge \omega^{s_{n-r_{1}}}>.
$$

LEMMA B.5. If $W \subset V$ is a q-dimensional subspace transverse to $I^{1}$, and for some $k, 1 \leq k \leq n-r_{1}, I=\left\langle I^{1}, I^{2}, \ldots I^{k}\right\rangle$, then $I_{A}=$ $<A^{1}, A^{2}, \ldots A^{k}>$.

Proof. We start by assuming that $2 \leq k \leq n-r_{1}$ and use the generators from Corollary B.1. Choose $l$ satisfying $k<l \leq n-r_{1}$. The condition that $I^{1}, \ldots, I^{k}$ generate $I$ imply that for all $l$ satisfying $k<l \leq n-r_{1}$ there exists $\kappa_{v_{m}}^{u_{l}} \in \Lambda^{l-m}\left(V^{*}\right), m=1 \ldots k-1$ such that

$A_{s_{1} \ldots s_{l}}^{u_{l}} \omega^{s_{1}} \wedge \ldots \wedge \omega^{s_{l}}=\kappa_{v_{k}}^{u_{l}} \wedge A_{s_{1} \ldots s_{k}}^{v_{k}} \omega^{s_{1}} \wedge \ldots \wedge \omega^{s_{k}}+\ldots+\kappa_{v_{2}}^{u_{l}} \wedge A_{s_{1} s_{2}}^{v_{2}} \omega^{s_{1}} \wedge \omega^{s_{2}}$.

This immediately implies that $A_{s_{1} \ldots s_{l}}^{u_{l}} \omega^{s_{1}} \wedge \ldots \wedge \omega^{s_{l}}$ may be discarded as a generator for $I_{A}$. So $A^{l} \subset<A^{1}, \ldots A^{k}>$, and $I_{A}=\left\langle A^{1}, \ldots, A^{k}\right\rangle$. The case $k=1$ is easy.

COROLLARY B.2. Starting with the hypothesis of Lemma B.5, let

$$
\begin{aligned}
\left\{A_{s_{1} s_{2}}^{w_{2}} \omega^{s_{1}} \wedge \omega^{s_{2}}\right\} \subset & \left\{A_{s_{1} s_{2}}^{u_{2}} \omega^{s_{1}} \wedge \omega^{s_{2}}\right\}, \ldots \\
& \left\{A_{s_{1} \ldots s_{l}}^{w_{k}} \omega^{s_{1}} \wedge \ldots \wedge \omega^{s_{k}}\right\} \subset\left\{A_{s_{1} \ldots s_{l}}^{u_{k}} \omega^{s_{1}} \wedge \ldots \wedge \omega^{s_{k}}\right\}
\end{aligned}
$$

be subsets which project to basis for $I^{2} \bmod I^{1}, \ldots, I^{k} \bmod I^{k-1}$ then

$$
I=<\eta^{i}, \theta^{a_{1}}, A_{s_{1} s_{2}}^{w_{2}} \omega^{s_{1}} \wedge \omega^{s_{2}}, \ldots, A_{s_{1} \ldots s_{k}}^{w_{k}} \omega^{s_{1}} \wedge \ldots \wedge \omega^{s_{k}}>
$$

and

$$
I_{A}=<\theta^{a_{1}}, A_{s_{1} s_{2}}^{w_{2}} \omega^{s_{1}} \wedge \omega^{s_{2}}, \ldots, A_{s_{1} \ldots s_{k}}^{w_{k}} \omega^{s_{1}} \wedge \ldots \wedge \omega^{s_{k}}>.
$$

and these generators are a minimal set. 


\section{References}

Anderson I.M, Fels M.E. Hyperbolic Exterior Differential Systems with Symmetry. preparation.

Anderson I.M, Fels M.E., Torre C.G. Group invariant solutions without transversality. Comm. Math. Phys., 212(3):653-686, 2000.

Anderson I.M., Kamran N., Olver P.J. Internal, external and generalized Symmetries. Adv. Math., 100(1):53-100, 1992.

Bluman G., Anco S. Symmetry and Integration Methods for Differential Equations, Springer-Verlag, 2002.

Symmetries and differential equations. Applied Mathematical Sciences SpringerVerlag,81, 1989.

Bluman G., Kumei S. Symmetries and differential equations. Applied Mathematical Sciences Springer-Verlag,81, 1989.

Bryant R.L.,Chern S.S.,Gardner R.B.,Goldschmidt H.L.,Griffiths P.A. Exterior Differential Systems. Spinger-Verlag, 1991.

Bryant R.L.,Griffiths P.A. Characteristic cohomology of differential systems. II. Conservation laws for a class of parabolic equations. Duke Math. J., 78,531-676, 1995.

Bryant R.L.,Griffiths P.A.,Hsu L. Hyperbolic Exterior Differential Systems and their Conservation Laws, Part I. Selecta Mathematica, New Series, 1,21-112, 1995.

Fels M.E. Integrating Scalar Ordinary Differential Equations with Symmetry Revisited. In preparation.

Freed D.S., Uhlenbeck K.K. Geometry and Quantum Field Theory. American Mathematics Society, 1995.

Itskov V. Orbit reduction of contact ideals. Contemp. Math., 285,171-181, 2001.

Krasilcshchik I.S., Vinogradov A.M. Nonlocal symmetries and the theory of coverings Local symmetries and conservation laws. Acta Appl. Math., 2,21-78,1984.

Kumpera A. On the Lie and Cartan Theory of Invariant Differential Systems. J. Math. Sci. Univ. Tokyo., 6,229-314,1999.

Olver P.J. Applications of Lie Groups to Differential Equations Springer-Verlag, 1998.

Ovsiannikov L.V. Group analysis of differential equations. Academic Press , 1982. 
new_tut_f.tex; 26/09/2004; $12: 45 ;$ p.32 